\title{
The evolution of a slender non-axisymmetric drop in an extensional flow
}

\author{
By P. D. HOWELL AND M. SIEGEL $L^{2}$ \\ ${ }^{1}$ OCIAM, Mathematical Institute, 24-29 St Giles', Oxford OX1 3LB, UK \\ ${ }^{2}$ Department of Mathematical Sciences, NJIT, University Heights, \\ Newark, NJ 07102-1982, USA
}

(Received 6 August 2004)

An asymptotic method for analysing slender non-axisymmetric drops, bubbles and jets in a general straining flow is developed. The method relies on the slenderness of the geometry to reduce the three-dimensional equations to a sequence of weakly coupled, quasitwo-dimensional Stokes flow problems for the cross-sectional evolution. Exact solution techniques for the flow outside a bubble in two-dimensional Stokes flow are generalised to solve for the transverse flow field, allowing large non-axisymmetric deformations to be described. A generalisation to the case where the interior of the contains a slightly viscous fluid is also presented.

Our method is used to compute steady non-axisymmetric solution branches for inviscid bubbles and slightly viscous drops. We also present unsteady numerical solutions showing how the eccentricity of the cross-section adjusts to a non-axisymmetric external flow. Finally, we use our theory to investigate how the pinch-off of a jet of relatively inviscid fluid is affected by a two-dimensional straining cross-flow. 


\section{Introduction}

This paper concerns the evolution of a slender bubble, drop or jet in an external fluid of much higher viscosity. There are two principle motivations for this study. The first is the famous work of Taylor (1934) on the behaviour of a relatively inviscid drop when subjected to a straining flow in a much more viscous fluid (see also the review article by Stone 1994). Two-dimensional models of an inviscid bubble in a viscous straining flow have received much attention, mainly due to the existence of exact solutions which result from the application of complex variable methods. The first steady solutions were obtained by Richardson (1968) (generalised by Antanovskii 1996) and later extended to include time dependence by Antanovskii (1994) and Tanveer \& Vasconcelos (1995). Other authors (Taylor 1964; Buckmaster 1972, 1973; Acrivos \& Lo 1978) have analysed the axisymmetric version of the problem, although the external flow used by Taylor (1934) was clearly two-dimensional. This shortcoming was addressed by Hinch \& Acrivos (1979), who viewed the problem as a perturbation of the axisymmetric version. Our method, however, gives a general approach for analysing large non-axisymmetric deformations of a bubble in a straining flow, whether two-dimensional, axisymmetric, or otherwise.

The second focus of our study is the break-up of a slender fluid jet inside an external, more viscous, fluid. The axisymmetric version of this scenario has previously been considered, for example, by Zhang \& Lister (1999), Doshi et al. (2003), and Sierou \& Lister (2003). We generalise these studies to cases in which the jet is not axisymmetric and may be driven by a transverse straining flow.

Our approach is similar in principle to that applied by Cummings \& Howell (1999) to slender viscous fibres. The slenderness of the geometry is used to reduce the threedimensional equations to a sequence of weakly-coupled quasi-two-dimensional problems, each of which closely resembles two-dimensional Stokes flow outside a bubble. Hence 
we simply have to apply the exact two-dimensional Stokes flow solutions obtained by Tanveer \& Vasconcelos (1995) to our quasi-two-dimensional problem.

Although the method described in Tanveer \& Vasconcelos (1995) allows a great number of two-dimensional bubble shapes to be described, in this paper we concentrate on the simple case in which the bubble has an elliptical cross-section, so we can also solve for the flow of the fluid inside the bubble. We therefore obtain a new system of equations governing the average radius and eccentricity of each cross-section and the internal pressure. Our equations apply to any slender bubble, drop or jet, provided the internal viscosity is significantly less than that outside. We present numerical solutions that illustrate important differences from the axisymmetric versions previously analysed.

In section 2 , we state the main governing equations and boundary conditions for the flow of a viscous fluid outside an inviscid bubble. In section 3, we use the assumed slenderness of the geometry to reduce the general three-dimensional equations to a quasi-twodimensional problem, which is analysed by generalising the complex-variable methods employed by Tanveer \& Vasconcelos (1995). In section 4, we apply these methods to the special case in which the bubble cross-section is assumed to be elliptical. In this case, the problem reduces to two partial differential equations for the average radius and eccentricity of the bubble. The steady-state solution of these equations is found and shown to be a small perturbation of the axisymmetric solution, in agreement with Hinch \& Acrivos (1979). Unsteady solutions are obtained numerically, allowing us to investigate how the shape of the cross-section adjusts to a non-axisymmetric external flow.

In section 5, we suppose the viscosity of the fluid inside the bubble is small but nonzero. In this case, the velocity inside the bubble satisfies a Dirichlet problem which, fortunately, can be solved exactly if the cross-section is elliptical. We thus obtain a new system of partial differential equations governing the evolution of a relatively inviscid bubble 


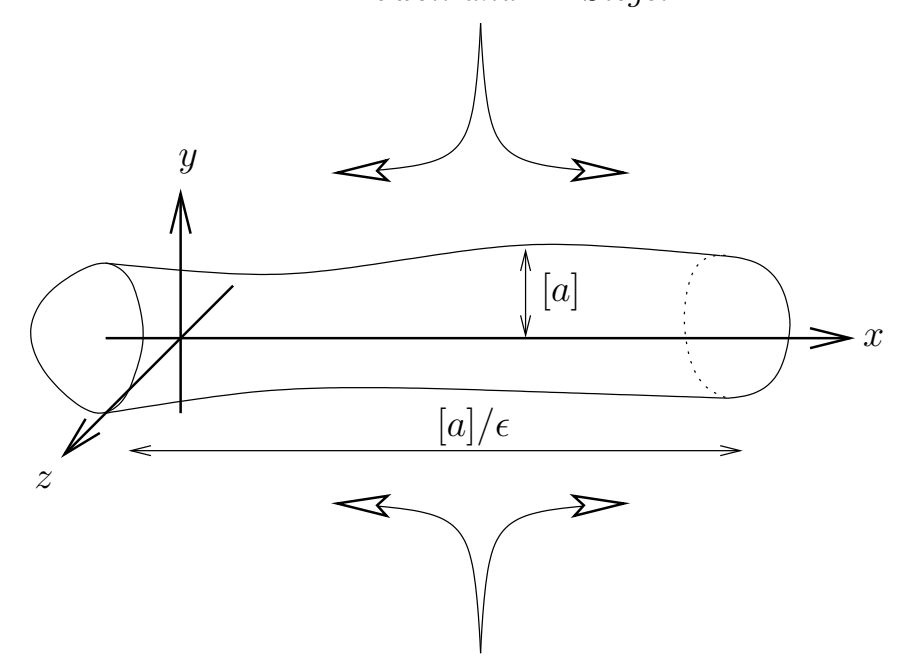

FiguRE 1. Schematic of a slender bubble in an extensional flow.

in a non-axisymmetric external Stokes flow. Interestingly, the steady solutions of these equations are shown to differ markedly from those given in Acrivos \& Lo (1978) for the axisymmetric problem. The difference between the two solutions is due to the existence of an alternative branch of axisymmetric solutions found previously by Buckmaster (1973). We also solve the unsteady problem numerically using a modal expansion technique similar to that employed by Hinch \& Acrivos (1980); Sherwood (1984).

The governing equations derived here apply to any extensional Stokes flow containing a slender drop of inviscid or much-less-viscous fluid. In section 6, we apply the theory to a jet of relatively inviscid fluid subject to a two-dimensional straining cross-flow. The axisymmetric version of this problem has previously been analysed by, for example, Zhang \& Lister (1999) and Sierou \& Lister (2003), who found that canonically the jet pinches off in finite time. We find that the jet may not pinch if a sufficiently large transverse straining flow is applied, instead being squashed by the external flow. 


\section{Problem statement}

Inertia is neglected throughout this paper. Our starting point is therefore the Stokes equations,

$$
\boldsymbol{\nabla} p=\mu \nabla^{2} \boldsymbol{u}, \quad \boldsymbol{\nabla} \cdot \boldsymbol{u}=0,
$$

where $p, \boldsymbol{u}$ and $\mu$ are the fluid pressure, velocity and viscosity respectively. We take as our unperturbed flow the exact solution of these equations given by

$$
\boldsymbol{u}=\left(\begin{array}{c}
\alpha x \\
\beta y \\
-(\alpha+\beta) z
\end{array}\right), \quad p=p_{\text {out }},
$$

where $\alpha, \beta$ and $p_{\text {out }}$ are constants. This pure straining or "extensional" flow is of particular relevance to many slow viscous flow problems in, for example, the glass industry (see Howell 1994). It has as special cases the two-dimensional stagnation-point flow used experimentally by Taylor (1934) $(\beta=-\alpha)$ and the axisymmetric extensional flow analysed by Taylor (1964) and subsequent authors $(\beta=-\alpha / 2)$.

We now wish to determine the evolution of an inviscid bubble placed in such a flow. For the moment we assume nothing about the shape of the bubble except that it is slender, by which we mean that its length-scale in (say) the $x$-direction is much longer than that in the $y$ - and $z$-directions.Thus, if a typical bubble radius is $[a]$, then a typical length along the bubble is $[a] / \epsilon$, where $\epsilon \ll 1$; see the definition sketch in figure 1 . In the neighbourhood of the bubble, we therefore non-dimensionalise (2.1) according to the scalings

$$
\begin{gathered}
x=\frac{[a]}{\epsilon} x^{\prime}, \quad y=[a] y^{\prime}, \quad z=[a] z^{\prime}, \\
u=\frac{\sigma}{\epsilon \mu} u^{\prime}, \quad v=\frac{\sigma}{\mu} v^{\prime}, \quad w=\frac{\sigma}{\mu} w^{\prime}, \\
p=p_{\text {out }}+\frac{\sigma}{[a]} p^{\prime},
\end{gathered}
$$


where the primes denote dimensionless quantities and $\sigma$ is the surface tension on the bubble surface. The non-dimensionalised Stokes equations then take the form (dropping primes)

$$
\begin{gathered}
u_{x}+v_{y}+w_{z}=0, \\
\epsilon^{2} p_{x}=\epsilon^{2} u_{x x}+u_{y y}+u_{z z}, \\
p_{y}=\epsilon^{2} v_{x x}+v_{y y}+v_{z z}, \\
p_{z}=\epsilon^{2} w_{x x}+w_{y y}+w_{z z} .
\end{gathered}
$$

Suppose the surface of the bubble is given by $G(x, y, z, t)=0$. Then on this surface we impose the kinematic boundary condition,

$$
G_{t}+u G_{x}+v G_{y}+w G_{z}=0,
$$

and a balance between viscous stresses, surface tension and the pressure $p_{\text {in }}$ inside the bubble. We denote by $\mathcal{P}$ the dimensionless effective pressure inside the bubble:

$$
\mathcal{P}=\frac{[a]}{\sigma}\left(p_{\text {in }}-p_{\text {out }}\right) .
$$

Then, if $\kappa$ is the curvature (made dimensionless with $[a]^{-1}$ ), the stress balance on the bubble surface $G(x, y, z, t)=0$ takes the form

$$
\left(\begin{array}{c}
\epsilon^{2}\left(-p+2 u_{x}\right) G_{x}+\left(u_{y}+\epsilon^{2} v_{x}\right) G_{y}+\left(u_{z}+\epsilon^{2} w_{x}\right) G_{z} \\
\left(u_{y}+\epsilon^{2} v_{x}\right) G_{x}+\left(-p+2 v_{y}\right) G_{y}+\left(v_{z}+w_{y}\right) G_{z} \\
\left(u_{z}+\epsilon^{2} w_{x}\right) G_{x}+\left(v_{z}+w_{y}\right) G_{y}+\left(-p+2 w_{z}\right) G_{z}
\end{array}\right)=(-\mathcal{P}+\kappa)\left(\begin{array}{c}
\epsilon^{2} G_{x} \\
G_{y} \\
G_{z}
\end{array}\right) .
$$

The problem is closed by prescribing the flow at infinity, in our case the extensional flow (2.2). For convenience, we set

$$
\delta=\frac{\beta}{\alpha}+\frac{1}{2},
$$


so that $\delta$ measures the asymmetry of the outer flow. In particular, $\delta=0$ corresponds to the outer flow being axisymmetric while $\delta= \pm 1 / 2$ gives a two-dimensional outer flow. Hence the required behaviour is

$$
\left.\begin{array}{l}
u \sim \mathrm{C} x \\
v \sim \mathrm{C}\left(\delta-\frac{1}{2}\right) y \\
w \sim-\mathrm{C}\left(\delta+\frac{1}{2}\right) z \\
p \rightarrow 0
\end{array}\right\} \text { as } \sqrt{y^{2}+z^{2}} \rightarrow \infty,
$$

where the capillary number $\mathrm{C}$ is defined by

$$
\mathrm{C}=\frac{\mu \alpha[a]}{\sigma} .
$$

\section{Problem simplification}

\subsection{Leading-order equations}

We seek solutions to this problem in the form of asymptotic expansions in powers of the slenderness parameter $\epsilon$, typically

$$
u \sim u_{0}+\epsilon^{2} u_{1}+\epsilon^{4} u_{2}+\cdots .
$$

The problem for $u_{0}$ implies that we must take

$$
u_{0}=\mathrm{C} x
$$

and the problem for the leading-order cross-flow is

$$
v_{0_{y}}+w_{0_{z}}=-\mathrm{C}, \quad p_{0_{y}}=v_{0_{y y}}+v_{0_{z z}}, \quad p_{0_{z}}=w_{0_{y y}}+w_{0_{z z}},
$$

with

$$
\left.\begin{array}{l}
G_{0_{t}}+\mathrm{C} x G_{0_{x}}+v_{0} G_{0_{y}}+w_{0} G_{0_{z}}=0 \\
\left(-p_{0}+2 v_{0_{y}}\right) G_{0_{y}}+\left(v_{0_{z}}+w_{0_{y}}\right) G_{0_{z}}=\left(-\mathcal{P}+\kappa_{0}\right) G_{0_{y}} \\
\left(v_{0_{z}}+w_{0_{y}}\right) G_{0_{y}}+\left(-p_{0}+2 w_{0_{z}}\right) G_{0_{z}}=\left(-\mathcal{P}+\kappa_{0}\right) G_{0_{z}}
\end{array}\right\} \text { on } G_{0}(x, y, z, t)=0,
$$


and

$$
\left.\begin{array}{l}
v_{0} \sim \mathrm{C}\left(\delta-\frac{1}{2}\right) y \\
w_{0} \sim-\mathrm{C}\left(\delta+\frac{1}{2}\right) z \\
p_{0} \rightarrow 0
\end{array}\right\} \text { as } y^{2}+z^{2} \rightarrow \infty .
$$

\subsection{Complex-variable methods}

We now transform the problem into a modified version of the purely two-dimensional problem studied by Tanveer \& Vasconcelos (1995) via the substitutions

$$
v_{0}=-\frac{1}{2} \mathrm{C} y+\tilde{v}, \quad w_{0}=-\frac{1}{2} \mathrm{C} z+\tilde{w}, \quad p_{0}=\mathcal{P}-\mathrm{C}+\tilde{p} .
$$

The result is the system

$$
\tilde{v}_{y}+\tilde{w}_{z}=0, \quad \tilde{p}_{y}=\tilde{v}_{y y}+\tilde{v}_{z z}, \quad \tilde{p}_{z}=\tilde{w}_{y y}+\tilde{w}_{z z},
$$

with the behaviour at infinity given by

$$
\tilde{p} \sim \mathrm{C}-\mathcal{P}, \quad \tilde{v} \sim \delta \mathrm{C} y, \quad \tilde{w} \sim-\delta \mathrm{C} z, \quad \text { as } y^{2}+z^{2} \rightarrow \infty
$$

The stress conditions are transformed to

$$
\left.\begin{array}{l}
\left(-\tilde{p}+2 \tilde{v}_{y}\right) G_{0_{y}}+\left(\tilde{v}_{z}+\tilde{w}_{y}\right) G_{0_{z}}=\kappa_{0} G_{0_{y}} \\
\left(\tilde{v}_{z}+\tilde{w}_{y}\right) G_{0_{y}}+\left(-\tilde{p}+2 \tilde{w}_{z}\right) G_{0_{z}}=\kappa_{0} G_{0_{z}}
\end{array}\right\} \text { on } G_{0}(x, y, z, t)=0
$$

Note that, as in Cummings \& Howell (1999), the leading-order curvature $\kappa_{0}$ of the free surface is simply the curvature of the boundary of each cross-section; the dependence on $x$ only comes in at higher order. Therefore (3.7), (3.8) and (3.9) are identical to the equations and boundary conditions for purely two-dimensional Stokes flow outside a bubble with an extensional flow at infinity, though the kinematic condition on $G_{0}(x, y, z, t)=0$ is altered to

$$
G_{0_{t}}+\tilde{v} G_{0_{y}}+\tilde{w} G_{0_{z}}=\mathrm{C}\left(-x G_{0_{x}}+\frac{1}{2} y G_{0_{y}}+\frac{1}{2} z G_{0_{z}}\right) .
$$

The right-hand side of (3.10) is identically zero for the purely two-dimensional problem 
(which is recovered if there is no flow at infinity so $\mathrm{C}=0$ ). In this case, a procedure for solving the problem in terms of a time-dependent conformal map from the unit disc onto the outside of the bubble has been given by Tanveer \& Vasconcelos (1995). We will now generalise their analysis to the case in which the right-hand side of (3.10) is not identically zero.

As a result of (3.7), we may employ a streamfunction $\psi$, in terms of which $\tilde{v}$ and $\tilde{w}$ are given by $\psi_{z}$ and $-\psi_{y}$ respectively. Elimination of $\tilde{p}$ from (3.7) reveals that $\psi$ satisfies the two-dimensional biharmonic equation, and hence may be expressed in the form

$$
\psi=\Im[\overline{\mathcal{Z}} f(\mathcal{Z})+g(\mathcal{Z})]
$$

where $f$ and $g$ are analytic functions of $\mathcal{Z}=y+\mathrm{i} z$ in the fluid region. From (3.8), we know that the velocity field should look like

$$
\tilde{v}+\mathrm{i} \tilde{w} \sim \delta \mathrm{C} \overline{\mathcal{Z}}+\frac{m}{2 \pi \overline{\mathcal{Z}}}+\frac{D}{\mathcal{Z}}+\frac{\bar{D} \mathcal{Z}}{\overline{\mathcal{Z}}^{2}}+O\left(\frac{1}{\mathcal{Z}^{2}}\right) \quad \text { as } \mathcal{Z} \rightarrow \infty,
$$

where the terms in this expansion correspond to the imposed extensional flow, a mass source (this will lead to evolution in the cross-sectional area of the bubble) and a twodimensional stresslet respectively. From this, the behaviour of $f$ and $g$ can be deduced:

$$
\left.\begin{array}{l}
f(\mathcal{Z}) \sim \frac{\mathrm{C}-\mathcal{P}}{4} \mathcal{Z}+B+\frac{D}{\mathcal{Z}}+O\left(\frac{1}{\mathcal{Z}^{2}}\right) \\
g^{\prime}(\mathcal{Z}) \sim \delta \mathrm{C} \mathcal{Z}+\bar{B}+\frac{m}{2 \pi \mathcal{Z}}+O\left(\frac{1}{\mathcal{Z}^{2}}\right)
\end{array}\right\} \text { as } \mathcal{Z} \rightarrow \infty,
$$

where $m, B$ and $D$ are (as yet) arbitrary functions of $x$ and $t$.

The stress condition (3.9) leads to the following relation between $f$ and $g$ on the bubble surface (Tanveer \& Vasconcelos 1995, equation 30)

$$
f(\mathcal{Z})+\mathcal{Z} \bar{f}^{\prime}(\overline{\mathcal{Z}})+\bar{g}^{\prime}(\overline{\mathcal{Z}})=-\frac{1}{2} \mathrm{i} \mathcal{Z}_{s}
$$

where $\mathcal{Z}_{s}$ represents differentiation with respect to arc length around the boundary of the bubble cross-section. This means that the transverse velocity components on the surface 
of the bubble are given by

$$
\tilde{v}+\mathrm{i} \tilde{w}=-\frac{1}{2} \mathrm{i} \mathcal{Z}_{s}-2 f(\mathcal{Z})
$$

We postpone discussion of the kinematic boundary condition (3.10) until we have set up our description of the shape of the bubble in terms of an $x$ - and $t$-dependent conformal map in the following paragraph.

Now we suppose that the exterior of the cross-section of the bubble is the image of the unit disc in the $\zeta$-plane under the conformal map $\mathcal{Z}=\Omega(\zeta ; x, t)$. As in Tanveer \& Vasconcelos (1995), we assume that the origin in the $\zeta$-plane is mapped to infinity in the $\mathcal{Z}$-plane, so that $\Omega(\zeta)$ is of the general form

$$
\Omega(\zeta ; x, t)=\frac{a(x, t)}{\zeta}+h(\zeta ; x, t)
$$

where $h(\zeta ; x, t)$ is analytic in $|\zeta| \leq 1$. Henceforth the dependence of $\Omega$ on $x$ and $t$ will be implicit; we shall denote $\Omega(\zeta ; x, t)$ by $\Omega(\zeta)$ and $\Omega_{\zeta}(\zeta ; x, t)$ by $\Omega^{\prime}(\zeta)$. We also introduce the notation

$$
F(\zeta)=f(\Omega(\zeta)), \quad G(\zeta)=g^{\prime}(\Omega(\zeta))
$$

Since $\mathcal{Z}_{s}$ is given in terms of $\Omega(\zeta)$ by

$$
\mathcal{Z}_{s}=\frac{\mathrm{i} \zeta \Omega^{\prime}(\zeta)}{\left|\Omega^{\prime}(\zeta)\right|} \text { on }|\zeta|=1
$$

substitution for the velocity components from (3.15) into the kinematic condition (3.10) gives

$$
\operatorname{Re}\left[\frac{\Omega_{t}(\zeta)+\mathrm{C} x \Omega_{x}(\zeta)+\frac{1}{2} \mathrm{C} \Omega(\zeta)+2 F(\zeta)}{\zeta \Omega^{\prime}(\zeta)}\right]=\frac{1}{2\left|\Omega^{\prime}(\zeta)\right|} \quad \text { on }|\zeta|=1
$$

Note that the second and third terms in the numerator on the left-hand side of (3.19) are not present in the two-dimensional theory of Tanveer \& Vasconcelos (1995). The righthand side of (3.19) is clearly non-analytic, but can nevertheless be decomposed into the 
sum of two functions, one analytic in $|\zeta|<1$ and one in $|\zeta|>1$, via Poisson's formula:

$$
\frac{1}{\left|\Omega^{\prime}(\zeta)\right|}=I(\zeta)+\bar{I}\left(\zeta^{-1}\right)
$$

where

$$
I(\zeta)=\frac{1}{4 \pi i} \oint_{|\omega|=1}\left(\frac{\omega+\zeta}{\omega-\zeta}\right) \frac{\mathrm{d} \omega}{\omega\left|\Omega^{\prime}(\omega)\right|} .
$$

Hence, by analytic continuation, (3.19) implies that

$$
\Omega_{t}(\zeta)+\mathrm{C} x \Omega_{x}(\zeta)+\frac{1}{2} \mathrm{C} \Omega(\zeta)+2 F(\zeta)=\zeta \Omega^{\prime}(\zeta) I(\zeta) \quad \text { on }|\zeta|<1
$$

We can then derive an expression for $G(\zeta)$ by substituting for $\mathcal{Z}_{s}$ from (3.18) and (3.20) into (3.14). The result is

$$
\begin{aligned}
\Omega^{\prime}(\zeta) G(\zeta) & =-\bar{\Omega}\left(\zeta^{-1}\right) F^{\prime}(\zeta) \\
& +\frac{1}{2} \Omega^{\prime}(\zeta)\left(\bar{\Omega}_{t}\left(\zeta^{-1}\right)+\mathrm{C} x \bar{\Omega}_{x}\left(\zeta^{-1}\right)+\frac{1}{2} \mathrm{C} \bar{\Omega}\left(\zeta^{-1}\right)+\zeta^{-1} \bar{\Omega}^{\prime}\left(\zeta^{-1}\right) I(\zeta)\right)
\end{aligned}
$$

which holds originally on $|\zeta|=1$ and, hence, on $|\zeta| \leq 1$ by analytic continuation.

This completes the formulation of the cross-flow problem in general terms. Our solution procedure in practice mirrors that of Tanveer \& Vasconcelos (1995): the first stage is to propose a conformal map $\mathcal{Z}=\Omega(\zeta)$ in which the coefficients are functions of $x$ and $t$ which are to be determined (a discussion of the kind of maps for which the procedure will be successful is given in Tanveer \& Vasconcelos 1995). Then (3.13) gives the required singularities in $F(\zeta)=f(\Omega(\zeta))$ and $G(\zeta)=g^{\prime}(\Omega(\zeta))$ as $|\zeta| \rightarrow 0$. These can be used to match the singularities on the left- and right-hand sides of (3.22) and (3.23), resulting in equations for the unknown coefficients in $\Omega(\zeta)$. 


\section{Bubbles with elliptical cross-section}

\subsection{Governing equations}

We now demonstrate how the procedure described above works in practice by performing it for the simple case in which the boundary of the bubble cross-section is an ellipse. We suppose the conformal map $\Omega(\zeta)$ takes the form

$$
\Omega(\zeta)=y^{*}+\mathrm{i} z^{*}+\frac{a}{\zeta}+b \mathrm{e}^{2 \mathrm{i} \phi} \zeta
$$

so that it maps the disc $|\zeta|<1$ to the exterior of an ellipse, centred on the point $\left(y^{*}, z^{*}\right)$, with principal radii $(a+b)$ and $(a-b)$, rotated anticlockwise through an angle $\phi$. Here $y^{*}, z^{*}, a, b$ and $\phi$ are all a priori unknown functions of $x$ and $t$.

Substitution into (3.13) gives the leading-order behaviour of $F(\zeta)$ and $G(\zeta)$ as $|\zeta| \rightarrow 0$ :

$$
\begin{gathered}
F(\zeta) \sim \frac{a(\mathrm{C}-\mathcal{P})}{4 \zeta}+\left(\frac{(\mathrm{C}-\mathcal{P})\left(y^{*}+\mathrm{i} z^{*}\right)}{4}+B\right)+\left(\frac{b \mathrm{e}^{2 \mathrm{i} \phi}(\mathrm{C}-\mathcal{P})}{4}+\frac{D}{a}\right) \zeta+\cdots \\
G(\zeta) \sim \frac{\delta \mathrm{C} a}{\zeta}+\left(\delta \mathrm{C}\left(y^{*}+\mathrm{i} z^{*}\right)+\bar{B}\right)+\left(\delta \mathrm{Cbe}^{2 \mathrm{i} \phi}+\frac{m}{2 \pi a}\right) \zeta+\cdots
\end{gathered}
$$

We find $F(\zeta)$ in terms of $\Omega(\zeta)$ from $(3.22)$ :

$$
\begin{gathered}
2 F(\zeta)=\left(-\frac{a}{\zeta}+b \mathrm{e}^{2 \mathrm{i} \phi} \zeta\right) I(\zeta)-\left(y_{t}^{*}+\mathrm{C} x y_{x}^{*}+\frac{1}{2} \mathrm{C} y^{*}\right)-\mathrm{i}\left(z_{t}^{*}+\mathrm{C} x z_{x}^{*}+\frac{1}{2} \mathrm{C} z^{*}\right) \\
-\frac{1}{\zeta}\left(a_{t}+\mathrm{C} x a_{x}+\frac{1}{2} \mathrm{C} a\right)-\zeta \mathrm{e}^{2 \mathrm{i} \phi}\left(b_{t}+\mathrm{C} x b_{x}+\frac{1}{2} \mathrm{C} b+2 \mathrm{i} b\left(\phi_{t}+\mathrm{C} x \phi_{x}\right)\right) .
\end{gathered}
$$

Now we equate the behaviours of the left-hand and right-hand sides as $|\zeta| \rightarrow 0$. The behaviour of $I(\zeta)$ is

$$
I(\zeta) \sim I_{0}+I_{2} \zeta^{2}+\cdots
$$

where

$$
I_{0}=I(0)=\frac{1}{4 \pi} \int_{0}^{2 \pi} \frac{\mathrm{d} \theta}{\sqrt{a^{2}+b^{2}-2 a b \cos (2 \theta)}} .
$$

The coefficient of $\zeta^{-1}$ gives the equation

$$
a_{t}+\mathrm{C}(x a)_{x}+a\left(I_{0}-\frac{1}{2} \mathcal{P}\right)=0,
$$


while comparison of the constant terms gives an expression for $B$ :

$$
2 B+\frac{1}{2}(\mathrm{C}-\mathcal{P})\left(y^{*}+\mathrm{i} z^{*}\right)=-\left(y_{t}^{*}+\mathrm{C} x y_{x}^{*}+\frac{1}{2} \mathrm{C} y^{*}\right)-\mathrm{i}\left(z_{t}^{*}+\mathrm{C} x z_{x}^{*}+\frac{1}{2} \mathrm{C} z^{*}\right) .
$$

Finally, the coefficients of $\zeta$ lead to a formula for the constant $D$ :

$$
D+\frac{1}{2} a^{2} I_{2}=\frac{1}{2} a \mathrm{e}^{2 \mathrm{i} \phi}\left\{I_{0} b+\frac{1}{2}(\mathcal{P}-\mathrm{C}) b-b_{t}-\mathrm{C} x b_{x}-\frac{1}{2} \mathrm{C} b-2 \mathrm{i} b\left(\phi_{t}+\mathrm{C} x \phi_{x}\right)\right\} .
$$

Similarly, we now substitute the limiting behaviours of $F(\zeta)$ and $G(\zeta)$ into $(3.23)$ and compare the left- and right-hand sides. The real and imaginary parts of the $O\left(\zeta^{-3}\right)$ equation give

$$
(a b)_{t}+\mathrm{C}(x a b)_{x}+2 a b I_{0}=2 \delta \mathrm{C} a^{2} \cos (2 \phi)
$$

and

$$
b\left(\phi_{t}+\mathrm{C} x \phi_{x}\right)=-\delta \mathrm{C} a \sin (2 \phi) .
$$

Matching the $O\left(\zeta^{-2}\right)$ terms and substituting for $B$ from (4.7) results in equations for the centre-line of the bubble $\left(y^{*}(x, t), z^{*}(x, t)\right)$, namely

$$
y_{t}^{*}+\mathrm{C} x y_{x}^{*}=\left(\delta-\frac{1}{2}\right) \mathrm{C} y^{*}, \quad z_{t}^{*}+\mathrm{C} x z_{x}^{*}=-\left(\delta+\frac{1}{2}\right) \mathrm{C} z^{*} .
$$

These show that the cross-section is forced to move in such a way that the net uniform flow with respect to it is zero. Finally, a physically obvious mass-conservation equation is obtained from the coefficients of $\zeta^{-1}$ :

$$
\left(a^{2}-b^{2}\right)_{t}+\left(\mathrm{C} x\left(a^{2}-b^{2}\right)\right)_{x}=\frac{m}{\pi}
$$

(the cross-sectional area of the bubble is given by $\pi\left(a^{2}-b^{2}\right)$ ).

In summary, the centre-line equations (4.11) decouple and can be solved very simply (they clearly predict, for example, that a straight centre-line is stable for $|\delta|<1 / 2$ and unstable for $|\delta|>1 / 2$ ). Equations (4.6), (4.9) and (4.10) comprise a system for $a, b$ and $\phi$ which also involves the pressure $\mathcal{P}$ inside the bubble. For a two-dimensional bubble one might prescribe either $\mathcal{P}$ or the rate of change of area $m$ (Tanveer \& Vasconcelos 1995). 
Alternatively, if the bubble is compressible, the area and internal pressure are related by an equation of state (Pozrikidis 2001; Crowdy 2003). In our case, $\mathcal{P}$ is instead determined by a global constraint; we assume that the bubble is filled with incompressible inviscid fluid so its total volume is specified (as in Acrivos \& Lo 1978, for example).

For the remainder of this paper we assume that the rotation angle $\phi$ is identically zero and do not bother to solve for the bubble centre-line. Equations (4.6) and (4.9) may therefore be written in the form

$$
\begin{gathered}
a_{t}+\mathrm{C} x a_{x}+\left(\mathrm{C}-\frac{1}{2} \mathcal{P}\right) a+k(\nu)=0, \\
\nu_{t}+\mathrm{C} x \nu_{x}+(\mathcal{P}-\mathrm{C}) \nu=2 \delta \mathrm{C}
\end{gathered}
$$

where

$$
\nu=\frac{b}{a}, \quad k(\nu)=\frac{1}{4 \pi} \int_{0}^{2 \pi} \frac{\mathrm{d} \theta}{\sqrt{1+\nu^{2}-2 \nu \cos (2 \theta)}}=\frac{1}{\pi(1+\nu)} K\left(\frac{2 \sqrt{\nu}}{1+\nu}\right)
$$

and $K$ denotes the complete elliptic integral of the first kind (Gradshteyn \& Ryzhik 1994, page 907). Notice that the parameter $\nu$ depends only on the shape of the cross-section, not on its size; it is related to the eccentricity $e$ by

$$
e=\frac{2 \sqrt{\nu}}{1+\nu}
$$

\subsection{Steady state bubbles}

Since $k(0)=1 / 2,(4.13)$ reduces to the well-documented equation for an axisymmetric bubble if $\nu$ is set to zero. Buckmaster (1972) found that this equation admits steady-state solutions, analytic at $x=0$, only if $\mathcal{P}$ is of the form

$$
\mathcal{P}=2(2 n+1) \mathrm{C},
$$

for some positive integer $n$. If so, then the radius is given by

$$
a=\frac{1}{4 n \mathrm{C}}\left[1-\left(\frac{x}{\ell}\right)^{2 n}\right]
$$


where $\ell$ is the half-length of the bubble. Acrivos \& Lo (1978) showed that the only steady solution which is linearly stable and, therefore, likely to be observed in practice, is the one in which $n=1$.

Similarly, non-axisymmetric steady state solutions may be found which are analytic at $x=0$ for all $\mathcal{P}$ of the form $2(2 n+1) \mathrm{C}$, where $n$ is a positive integer, though only the case $n=1$ is linearly stable. This leads to the stable steady state:

$$
\mathcal{P}=6 \mathrm{C}, \quad \nu=\frac{2}{5} \delta, \quad \phi=0, \quad a=\frac{k(\nu)}{2 \mathrm{C}}\left[1-\left(\frac{x}{\ell}\right)^{2}\right] .
$$

Note that the behaviour of $k(\nu)$ when $\nu$ is small is

$$
k(\nu) \sim \frac{1}{2}+\frac{\nu^{2}}{8}+\frac{9 \nu^{4}}{128}+\cdots \quad \text { as } \nu \rightarrow 0,
$$

so that the axisymmetric solution found in Buckmaster (1972) is actually rather a good approximation to the non-axisymmetric solution provided $2 \delta / 5 \ll 1$. In particular, if the outer flow is two-dimensional (as in the original experiments of Taylor 1934), then $\delta=1 / 2, \nu=1 / 5$, and the difference between the solution for $a$ found by Buckmaster (1972) and the true solution (4.19) is just over one per cent. This conclusion was also reached by Hinch \& Acrivos (1979) by linearising about the axisymmetric solution.

\subsection{Unsteady bubbles}

To obtain unsteady bubble solutions, we have to specify initial conditions for $a$ and $\nu$ at $t=0$ along with the boundary condition and volume constraint

$$
a(\ell(t), t)=0, \quad \int_{0}^{\ell(t)} a(x, t)^{2}\left(1-\nu(x, t)^{2}\right) \mathrm{d} x=V=\text { const. }
$$

Along the characteristics $x \mathrm{e}^{-\mathrm{C} t}=$ const, the hyperbolic system (4.13), (4.14) reduces to two ordinary differential equations for $a$ and $\nu$. It is straightforward to show from (4.14) that, if $\nu$ is spatially uniform initially, then it remains so. Furthermore, even if $\partial \nu / \partial x$ is nonzero at $t=0$, it approaches zero as $t \rightarrow \infty$. We therefore suppose for simplicity that 
$\nu$ is a function only of $t$. In this case, (4.13) has the property that, if $a$ is initially an $n$ th-degree polynomial in $x$, then it remains so for all time. This property was previously exploited for the axisymmetric problem by Hinch (1980).

The problem is simplified by writing $\mathcal{P}$ in the form

$$
\mathcal{P}(t)=\mathrm{C}+\frac{\mathrm{d}^{2} / \mathrm{d} t^{2}\left(\mathrm{e}^{5 \mathrm{C} t} q(t)\right)}{\mathrm{d} / \mathrm{d} t\left(\mathrm{e}^{5 \mathrm{C} t} q(t)\right)}
$$

so the solution of (4.14) is

$$
\nu(t)=\frac{2 \delta \mathrm{C} q(t)}{\dot{q}(t)+5 \mathrm{C} q(t)},
$$

where $q(t)$ is chosen to satisfy the initial conditions

$$
q(0)=\frac{\nu(0)}{2 \delta \mathrm{C}}, \quad \dot{q}(0)=1-\frac{5 \nu(0)}{2 \delta}
$$

The solution of (4.13) may then be written as

$$
a(x, t)=\mathrm{e}^{2 \mathrm{C} t} \sqrt{\dot{q}(t)+5 \mathrm{C} q(t)}\left\{a_{0}\left(x \mathrm{e}^{-\mathrm{C} t}\right)-a_{0}\left(\ell(t) \mathrm{e}^{-\mathrm{C} t}\right)\right\}
$$

where $a_{0}(x)=a(x, 0)$ (in deriving(4.25), it is helpful first to differentiate (4.13) with respect to $x$ ) and

$$
\frac{k(\nu(t))}{\sqrt{\dot{q}(t)+5 \mathrm{C} q(t)}}=\mathrm{e}^{\mathrm{C} t}(\dot{\ell}(t)-\mathrm{C} \ell(t)) a_{0}^{\prime}\left(\ell(t) \mathrm{e}^{-\mathrm{C} t}\right)
$$

while the volume constraint leads to

$$
(\dot{q}(t)+5 \mathrm{C} q(t))\left(1-\nu(t)^{2}\right) \mathrm{e}^{5 \mathrm{C} t} \int_{0}^{\ell(t) \mathrm{e}^{-\mathrm{C} t}}\left(a_{0}(X)-a_{0}\left(\ell(t) \mathrm{e}^{-\mathrm{C} t}\right)\right)^{2} \mathrm{~d} X=V .
$$

Once the initial radius $a_{0}(x)$ is specified, (4.26) and (4.27) reduce to two ordinary differential equations for $\ell(t)$ and $q(t)$. We suppose that the bubble starts from a steady state corresponding to $\mathrm{C}=1, \mathcal{P}=6, \delta=0$, namely

$$
\nu(0)=0, \quad a_{0}(x)=\frac{1}{4}\left(1-x^{2}\right), \quad \ell(0)=1, \quad V=\frac{1}{30}
$$

The subsequent radius is given by

$$
a(x, t)=\frac{1}{4} \sqrt{\dot{q}(t)+5 \mathrm{C} q(t)}\left(\ell(t)^{2}-x^{2}\right)
$$




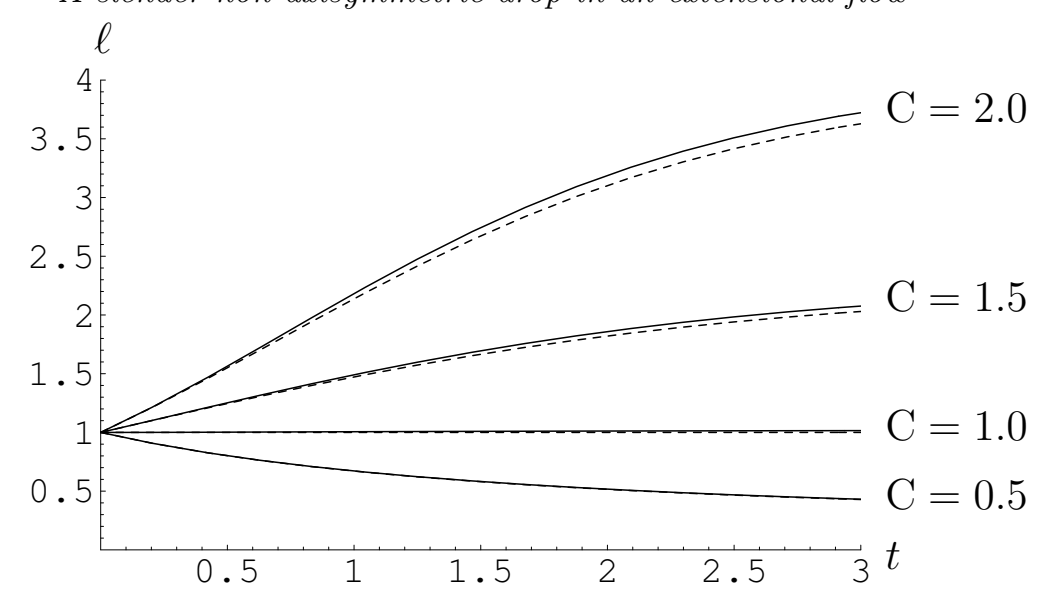

Figure 2. Bubble length $\ell$ versus time $t$ for $\delta=0.5$ (solid), $\delta=0$ (dashed) and various values of the capillary number $\mathrm{C}$.

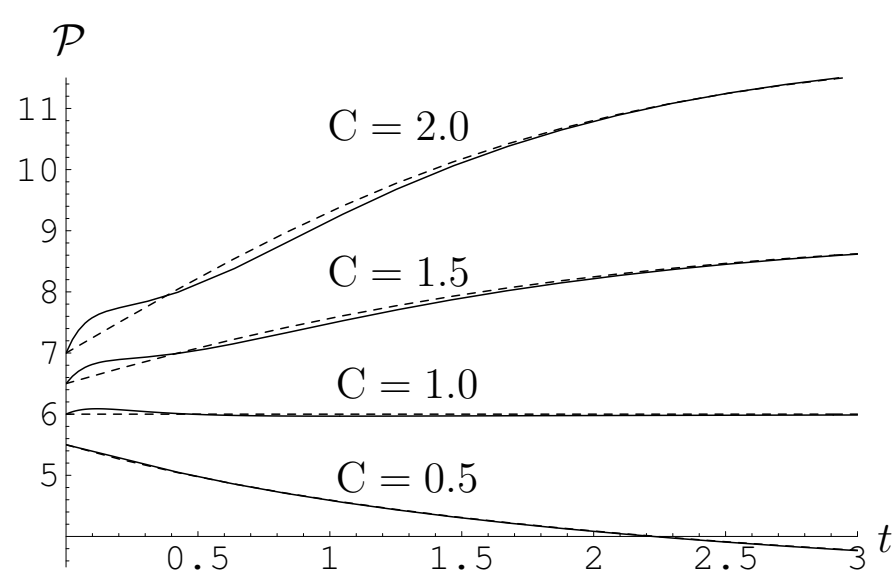

Figure 3. Bubble pressure $\mathcal{P}$ versus time $t$ for $\delta=0.5$ (solid), $\delta=0$ (dashed) and various values of the capillary number $\mathrm{C}$.

and the system (4.26), (4.27) becomes

$$
\begin{aligned}
& \frac{\mathrm{d} q}{\mathrm{~d} t}=-5 \mathrm{C} q+\frac{1}{2 \ell^{5}}\left(1+\sqrt{1+16 \delta^{2} \mathrm{C}^{2} \ell^{10} q^{2}}\right) \\
& \frac{\mathrm{d} \ell}{\mathrm{d} t}=\mathrm{C} \ell-\frac{2 \sqrt{2} \ell^{3 / 2}}{\sqrt{1+\sqrt{1+16 \delta^{2} \mathrm{C}^{2} \ell^{10} q^{2}}}} k\left(\frac{4 \delta \mathrm{C} \ell^{5} q}{1+\sqrt{1+16 \delta^{2} \mathrm{C}^{2} \ell^{10} q^{2}}}\right)
\end{aligned}
$$

to be solved subject to $\ell(0)=1, q(0)=0$.

In figures $2-4$, we illustrate some numerical solutions of (4.30) subject to $\delta=1 / 2$, so the initially axisymmetric bubble has to adjust to a two-dimensional flow, and various 


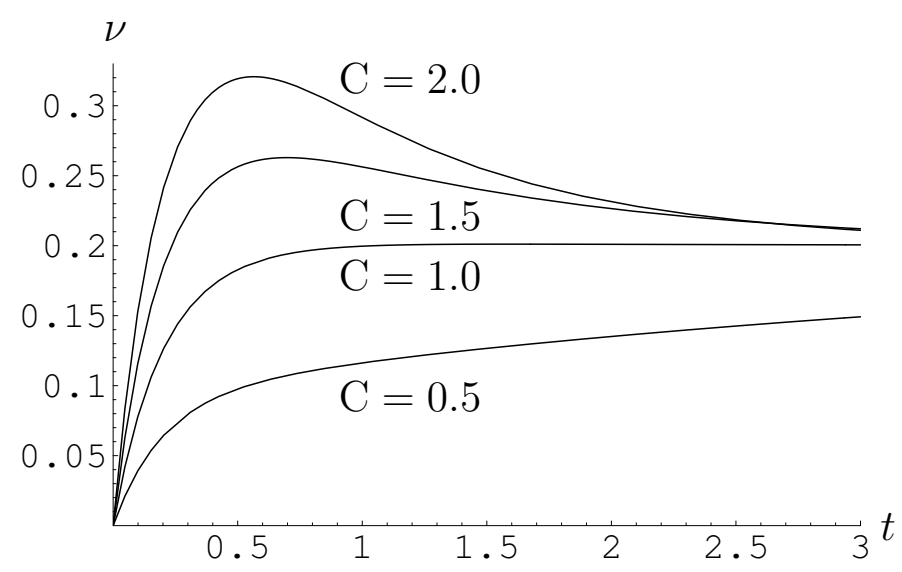

FiguRE 4. Cross-section eccentricity parameter $\nu$ versus time $t$ for various values of the capillary number C.

values of C. For comparison we also show as dashed lines the corresponding axisymmetric $(\nu=0)$ solutions (Hinch 1980), namely

$$
\ell=\frac{\mathrm{C}^{2} \mathrm{e}^{\mathrm{C} t}}{\left(\mathrm{C}-1+\mathrm{e}^{\mathrm{C} t / 2}\right)^{2}}, \quad \mathcal{P}=\mathrm{C}+\frac{5 \mathrm{C} \mathrm{e}^{\mathrm{C} t / 2}}{\mathrm{C}-1+\mathrm{e}^{\mathrm{C} t / 2}}
$$

In figure 2 we see how the bubble length $\ell$ adjusts monotonically from its initial value of 1 to its equilibrium value

$$
\ell(\infty)=\frac{25 \mathrm{C}^{2}}{96 k(1 / 5)^{2}} \approx 1.02 \mathrm{C}^{2},
$$

in each case staying close to the axisymmetric prediction. The corresponding behaviour of the bubble pressure $\mathcal{P}$ is shown in figure 3. Now there is an interesting deviation from the axisymmetric solution, even in the case $\mathrm{C}=1$ where the initial pressure $\mathcal{P}(0)=5+\mathrm{C}$ and equilibrium pressure $\mathcal{P}(\infty)=6 \mathrm{C}$ are equal. As shown in figure 4 , when $\mathrm{C}>1$, so the bubble length increases with $t, \nu$ significantly overshoots its equilibrium value of $1 / 5$. This evolution in the eccentricity of the cross-section forced by the asymmetry of the outer flow causes the initial transient growth in $\mathcal{P}$.

Finally we illustrate the behaviour of the principal radii $a(1+\nu)$ and $a(1-\nu)$ in figure 5 for $\mathrm{C}=0.5$ and $\mathrm{C}=2.0$. In either case, both evolve through a sequence of parabolas, 


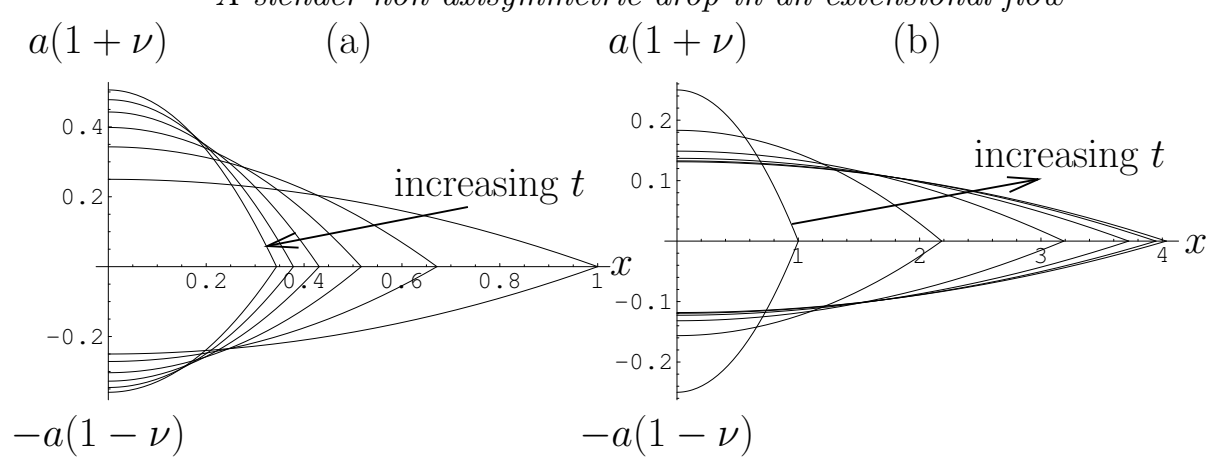

Figure 5. Principal radii $a(1+\nu)$ and $a(1-\nu)$ versus $x$ for $t=0,1,2,3,4,5$ and capillary number (a) $\mathrm{C}=0.5$, (b) $\mathrm{C}=2.0$.

with the length either increasing or decreasing according to whether $\mathrm{C}$ is greater or less than 1 . The initial shape is axisymmetric but the difference between the radii becomes noticeable as $\nu$ increases. Notice that the scalings for $a$ and $x$ are different, so the aspect ratio of the bubble appears deceptively small.

\section{A slightly viscous drop}

\subsection{General equations}

We now show how the analysis described thus far can be generalised to the case where the bubble (now referred to as a drop) contains slightly viscous fluid, with viscosity $\mu_{\mathrm{in}}$. As in Buckmaster (1973), we consider the scaling that yields the richest asymptotic balance, namely

$$
\frac{\mu_{\text {in }}}{\mu}=\epsilon^{2} \lambda
$$

where $\lambda$ is treated as an order-one constant. The non-dimensionalised equations (2.4) still hold outside the drop. We denote with overbars the pressure and velocity components for the fluid inside the drop, so that the dimensionless Stokes equations for that fluid take the form

$$
\bar{u}_{x}+\bar{v}_{y}+\bar{w}_{z}=0
$$




$$
\begin{gathered}
\text { P. D. Howell and M. Siegel } \\
\bar{p}_{x}=\lambda\left(\epsilon^{2} \bar{u}_{x x}+\bar{u}_{y y}+\bar{u}_{z z}\right), \\
\bar{p}_{y}=\epsilon^{2} \lambda\left(\epsilon^{2} \bar{v}_{x x}+\bar{v}_{y y}+\bar{v}_{z z}\right), \\
\bar{p}_{z}=\epsilon^{2} \lambda\left(\epsilon^{2} \bar{w}_{x x}+\bar{w}_{y y}+\bar{w}_{z z}\right) .
\end{gathered}
$$

On the surface of the drop, $G(x, y, z, t)=0$, we apply the kinematic conditions

$$
u=\bar{u}, \quad v=\bar{v}, \quad w=\bar{w}
$$

and

$$
G_{t}+u G_{x}+v G_{y}+w G_{z}=0=G_{t}+\bar{u} G_{x}+\bar{v} G_{y}+\bar{w} G_{z}
$$

We do not bother to write out the full stress balance equations on the drop surface but note that, since the viscosity inside the drop is an order of magnitude smaller than that outside, viscous stresses in the inner fluid play no role in the stress balance to leading order. The upshot is that the leading-order problem to be solved outside the drop is the same as that found in section 3.1, namely (3.3), (3.4) and (3.5); the only added complication is that the effective pressure inside the drop $\mathcal{P}=\bar{p}_{0}$, is now a function of $x$ as well as $t$. To determine $\mathcal{P}$, we must consider the leading-order solution inside the drop.

Equations (5.2c) and (5.2d) imply that the leading-order pressure $\mathcal{P}$ is independent of $y$ and $z$. The leading-order axial velocity may thus be written in the form

$$
\bar{u}_{0}=\mathrm{C} x-\lambda^{-1} \mathcal{P}_{x} U,
$$

where $U$ satisfies the canonical boundary-value problem

$$
\left.\begin{array}{ll}
U_{y y}+U_{z z}=-1 & \text { in } A_{0} \\
U=0 & \text { on } \partial A_{0}
\end{array}\right\}
$$

on the leading-order drop cross-section $A_{0}$. Hence $\bar{u}_{0}$ is given in principle as a function of the cross-sectional shape and $\mathcal{P}$. In general (5.6) must be solved separately for each $x$ and 
$t$; however, in the situations we have been considering in which the cross-section evolves through a one- or two-parameter family of shapes, we may expect to be able to determine $U$ in terms of these ( $x$ - and $t$-dependent) parameters. Notice that the conformal map $\Omega$ introduced in section 3.1 cannot in general be employed in the solution of (5.6), since it is not conformal inside the drop cross-section.

Once $U$ has been determined, the problem is closed by considering conservation of mass for the fluid inside the drop. As shown, for example, by Cummings \& Howell (1999), integration of (5.2a) over the cross-section and use of the kinematic boundary condition (5.4) gives rise to the equation

$$
\frac{\partial A_{0}}{\partial t}+\frac{\partial}{\partial x} \iint_{A_{0}} \bar{u}_{0} \mathrm{~d} y \mathrm{~d} z=0
$$

where $A_{0}$ is the leading-order area of the cross-section. Now we substitute for $\bar{u}_{0}$ from (5.5) and obtain

$$
A_{0_{t}}+\mathrm{C}\left(x A_{0}\right)_{x}=\lambda^{-1}\left(H A_{0}^{2} \mathcal{P}_{x}\right)_{x}
$$

where

$$
H=\frac{1}{A_{0}^{2}} \iint_{A_{0}} U \mathrm{~d} y \mathrm{~d} z
$$

is a functional that depends only on the shape of the cross-section, not on its size.

\subsection{A drop with elliptical cross-section}

The ellipse parameterised by (4.1) may be written in the form

$$
\frac{\tilde{y}^{2}}{(a+b)^{2}}+\frac{\tilde{z}^{2}}{(a-b)^{2}}=1,
$$

where

$$
y=y^{*}+\tilde{y} \cos \phi-\tilde{z} \sin \phi, \quad z=z^{*}+\tilde{z} \cos \phi+\tilde{y} \sin \phi
$$


Thus the problem (5.6) for $U$ may readily be solved to give

$$
U=\frac{\left(a^{2}-b^{2}\right)^{2}}{4\left(a^{2}+b^{2}\right)}\left(1-\frac{\tilde{y}^{2}}{(a+b)^{2}}-\frac{\tilde{z}^{2}}{(a-b)^{2}}\right)
$$

and the functional $H$ is hence given by

$$
H=\frac{a^{2}-b^{2}}{8 \pi\left(a^{2}+b^{2}\right)}=\frac{1-\nu^{2}}{8 \pi\left(1+\nu^{2}\right)} .
$$

To calculate the left-hand side of (5.8), we substitute $A_{0}=\pi\left(a^{2}-b^{2}\right)$ and use (4.13) and (4.9) to obtain

$$
A_{0_{t}}+\mathrm{C}\left(x A_{0}\right)_{x}=\pi\left(a^{2}+b^{2}\right)(\mathcal{P}-\mathrm{C})-\frac{2 \pi}{a}\left(a^{2}-b^{2}\right) k(\nu)-4 \pi \delta \mathrm{C}
$$

Thus, the problem to be solved is (4.13) and (4.14), with $\mathcal{P}$ determined from the coupled equation

$$
\left(\frac{\left(1-\nu^{2}\right)^{3}}{\left(1+\nu^{2}\right)} a^{4} \mathcal{P}_{x}\right)_{x}=8 \lambda a\left(\left(1+\nu^{2}\right) a(\mathcal{P}-\mathrm{C})-4 \delta \mathrm{C} \nu a-2\left(1-\nu^{2}\right) k(\nu)\right) .
$$

\subsection{Steady-state drops}

It is convenient to choose our scalings for $a$ and $x$ such that the dimensionless parameters $\mathrm{C}$ and $\lambda$ are both equal to one:

$$
[a]=\frac{\sigma}{\mu \alpha}, \quad \epsilon^{2}=\frac{\mu_{\text {in }}}{\mu} .
$$

In the steady state, the mass-conservation equation (5.8) may evidently be integrated with respect to $x$, thus reducing the problem to three first-order ordinary differential equations for $a(x), \nu(x)$ and $\mathcal{P}(x)$, namely

$$
\begin{aligned}
& x a^{\prime}=\left(\frac{1}{2} \mathcal{P}-1\right) a-k(\nu), \\
& x \nu^{\prime}=2 \delta+(1-\mathcal{P}) \nu \\
& \mathcal{P}^{\prime}=\frac{8 x\left(1+\nu^{2}\right)}{a^{2}\left(1-\nu^{2}\right)^{2}}
\end{aligned}
$$

We first consider the axisymmetric case $\delta=\nu=0$. Then the two remaining differential 
equations (5.17a) and (5.17c) admit the solution (given in Acrivos \& Lo 1978)

$$
a=\frac{4\left(\mathcal{P}_{0}-2\right)}{\mathcal{P}_{0}-6}\left(x^{2}-\ell^{2}\right), \quad \mathcal{P}=\mathcal{P}_{0}+\frac{\left(\mathcal{P}_{0}-6\right) x^{2}}{\ell^{2}-x^{2}}
$$

where $\mathcal{P}_{0}=\mathcal{P}(0)$ and $\ell$ is the dimensionless half-length of the drop, given by

$$
\ell=\frac{\sqrt{\mathcal{P}_{0}-6}}{2\left(\mathcal{P}_{0}-2\right)}
$$

There is a one-parameter family of such solutions, parameterised by $\mathcal{P}_{0}$, which evidently must exceed the value 6 (corresponding to the inviscid solution (4.19)) for the solutions to exist.

For each such value of $\mathcal{P}_{0}$, we can determine both $\ell$ and the corresponding dimensionless half-volume:

$$
V=\int_{0}^{\ell} a^{2} \mathrm{~d} x=\frac{4 \sqrt{\mathcal{P}_{0}-6}}{15\left(\mathcal{P}_{0}-2\right)^{3}}
$$

We thus infer the dimensional length $L$ and average radius $R$ of the drop from

$$
L=\frac{2 \sigma}{\mu \alpha} \sqrt{\frac{\mu}{\mu_{\mathrm{in}}}} \ell, \quad \frac{4}{3} R^{3}=2\left(\frac{\sigma}{\mu \alpha}\right)^{3} \sqrt{\frac{\mu}{\mu_{\mathrm{in}}}} V .
$$

In terms of these, we define a dimensionless drop length and strain rate by

$$
\mathcal{L}=\left(\frac{L}{R}\right)\left(\frac{\mu_{\mathrm{in}}}{\mu}\right)^{1 / 3}=2 \ell\left(\frac{2}{3 V}\right)^{1 / 3}, \quad \mathcal{A}=\left(\frac{\mu \alpha R}{\sigma}\right)\left(\frac{\mu_{\mathrm{in}}}{\mu}\right)^{1 / 6}=\left(\frac{3 V}{2}\right)^{1 / 3} .
$$

By eliminating $\mathcal{P}_{0}$, we obtain a functional relation between these two quantities, namely

$$
\mathcal{A}=\frac{\sqrt{5 \mathcal{L}}}{\sqrt{2}\left(10+\mathcal{L}^{3}\right)},
$$

which is plotted in figure 6 (solid line). This curve, equivalent to figure 1 in Taylor (1964) and figure 2 in Acrivos \& Lo (1978), indicates that, if the dimensionless strain rate exceeds a critical value

$$
\mathcal{A}=\frac{\sqrt{5}}{2^{1 / 3} 12} \approx 0.1479,
$$

then there is no steady solution and the drop must burst. For lower strain rates, there are 


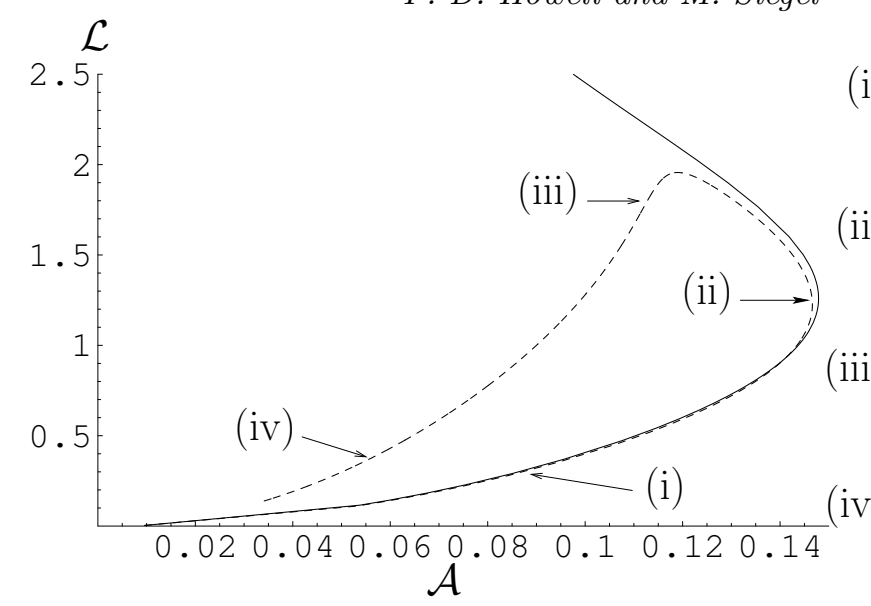

(i)

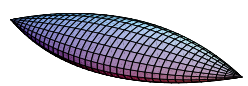

(ii)

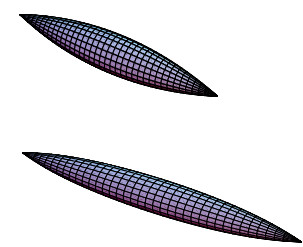

(iv)

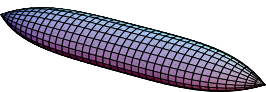

FiguRE 6. Bifurcation diagram of dimensionless drop length $\mathcal{L}$ versus dimensionless strain rate $\mathcal{A}$ for values of $\delta=0$ (solid) and $\delta=1 / 2$ (dashed).

two possible solutions, of which Acrivos \& Lo (1978) showed that only the lower branch is stable.

Now we turn our attention to the non-axisymmetric case. For each positive value of $\delta$, the three-dimensional system (5.17) also has a one-parameter family of solutions that are analytic at $x=0$, parameterised by $\mathcal{P}_{0}=\mathcal{P}(0)$. Although we do not have explicit solutions in this case, the equations are readily solved numerically. Provided $\mathcal{P}_{0}>6$, the radius $a$ reaches zero at a finite value of $x=\ell$. The corresponding dimensionless half-volume is now given by

$$
V=\int_{0}^{\ell} a^{2}\left(1-\nu^{2}\right) \mathrm{d} x .
$$

We again use (5.22) to plot the dimensionless drop length versus the dimensionless strain rate for $\delta=1 / 2$ in figure 6 (dashed line). The lower branches of the solid and dashed curves are very close and, in particular, increasing $\delta$ from 0 to $1 / 2$ reduces the critical strain rate by less than $1 \%$. We also show typical drop shapes at various points along the dashed curve. In none of them is the eccentricity of the cross-section noticeable to the naked eye. We can see, though, how the drop starts relatively short and fat on 


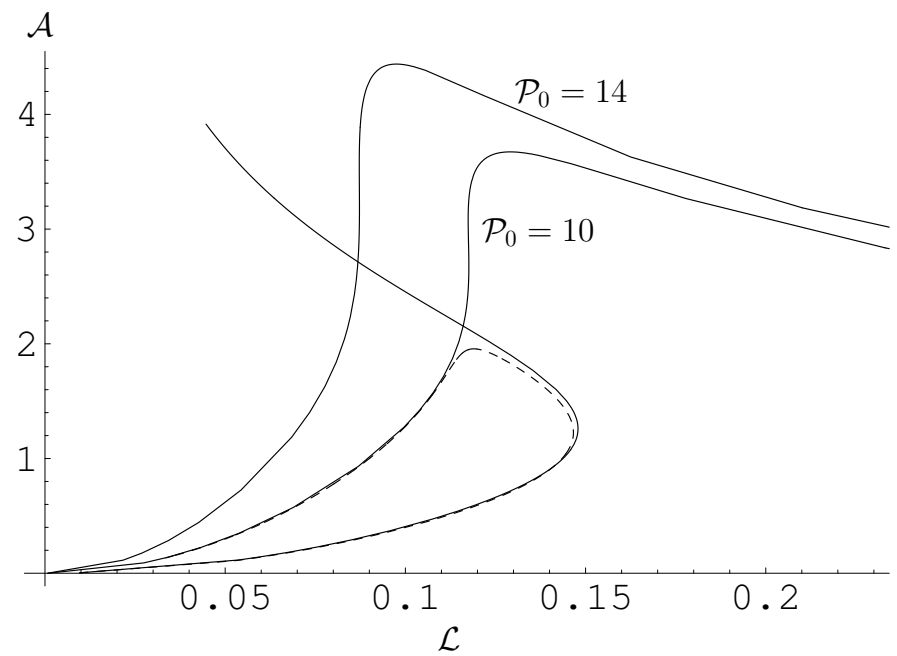

FiguRE 7. Dimensionless drop length versus dimensionless strain rate for $\delta=0$ (solid) and $\delta=1 / 2$ (dashed), also showing branches where $\mathcal{P}_{0}=10,14$.

the lower branch and becomes longer and thinner near the nose. On the upper branch, where the internal pressure is higher, the drops are more cigar-shaped, with radius almost uniform except near the tips where it decreases rapidly to zero.

Although the upper branches of the two solutions in figure 6 are unstable and, hence, of limited physical relevance, it is puzzling at first sight that they differ so markedly. The reason is that the $\delta=1 / 2$ curve latches onto a second branch of axisymmetric solutions noticed previously by Buckmaster (1973). If $\mathcal{P}_{0}$ is equal to one of the critical values $2(2 n+1)$ found by Buckmaster (1972), then $a$ need not be of the form (5.18). For example, analytic axisymmetric solution branches exist with

$$
\begin{array}{ll}
\mathcal{P}_{0}=10, & a \sim \frac{1}{8}-8 x^{2}+C_{1}\left(x^{4}+\frac{64}{3} x^{6}+\cdots\right), \\
\mathcal{P}_{0}=14, & a \sim \frac{1}{12}-6 x^{2}+C_{2}\left(x^{6}+72 x^{8}+\cdots\right),
\end{array}
$$

as $x \rightarrow 0$. These two branches, parameterised by $C_{j}$ instead of $\mathcal{P}_{0}$, are displayed in figure 7 . Here it is clear that the perturbed $\delta=1 / 2$ curve simply switches to the $\mathcal{P}_{0}=10$ branch at the point where it crosses the $\mathcal{P}_{0}=6$ branch. 
Interestingly, the new solution branches exist for super-critical strain rates. In this region they are presumably unstable, though, since they have the property that the drop length is a decreasing function of strain rate.

\subsection{Unsteady drops}

Next we present numerical solutions of the unsteady equations (4.13), (4.14) and (5.15). As above, we scale such that $\mathrm{C}$ and $\lambda$ are both equal to one. It is convenient to eliminate the internal pressure $\mathcal{P}$ using (4.13),

$$
\mathcal{P}=2+\frac{2}{a}\left(a_{t}+x a_{x}+k(\nu)\right)
$$

so that (5.15) and (4.14) may be written as the following system for $a$ and $\nu$ :

$$
\begin{gathered}
{\left[a^{2}\left(1-\nu^{2}\right)\right]_{t}+\left[x a^{2}\left(1-\nu^{2}\right)\right]_{x}=\left[\frac{a^{4}\left(1-\nu^{2}\right)^{3}}{4\left(1+\nu^{2}\right)}\left(\frac{a_{t}+x a_{x}+k(\nu)}{a}\right)_{x}\right]_{x},} \\
a\left(\nu_{t}+x \nu_{x}+\nu-2 \delta\right)+2 \nu\left(a_{t}+x a_{x}+k(\nu)\right)=0 .
\end{gathered}
$$

As the initial condition, we impose an equilibrium axisymmetric shape of dimensionless length $\mathcal{L}_{0}$ and strain rate $\mathcal{A}$ :

$$
a=\mathcal{A} \sqrt{\frac{5}{2 \mathcal{L}_{0}}}\left(1-\frac{4 x^{2}}{\mathcal{A}^{2} \mathcal{L}_{0}^{2}}\right), \quad \ell=\frac{\mathcal{A} \mathcal{L}_{0}}{2}, \quad \nu=0 \quad \text { at } t=0 .
$$

The volume of the drop is thus

$$
V=\int_{0}^{\ell} a^{2}\left(1-\nu^{2}\right) \mathrm{d} x=\frac{2 \mathcal{A}^{3}}{3}
$$

which remains constant throughout the subsequent evolution. By varying $\mathcal{A}$ and $\mathcal{L}_{0}$ we can start the drop at various points on the bifurcation diagram shown in figure 6 .

For the axisymmetric problem $\delta=\nu=0$, Buckmaster (1973) found the remarkable exact solution

$$
a(x, t)=\mathcal{A} \sqrt{\frac{5}{2 \mathcal{L}(t)}}\left(1-\frac{4 x^{2}}{\mathcal{A}^{2} \mathcal{L}(t)^{2}}\right), \quad \ell(t)=\frac{\mathcal{A L}(t)}{2},
$$



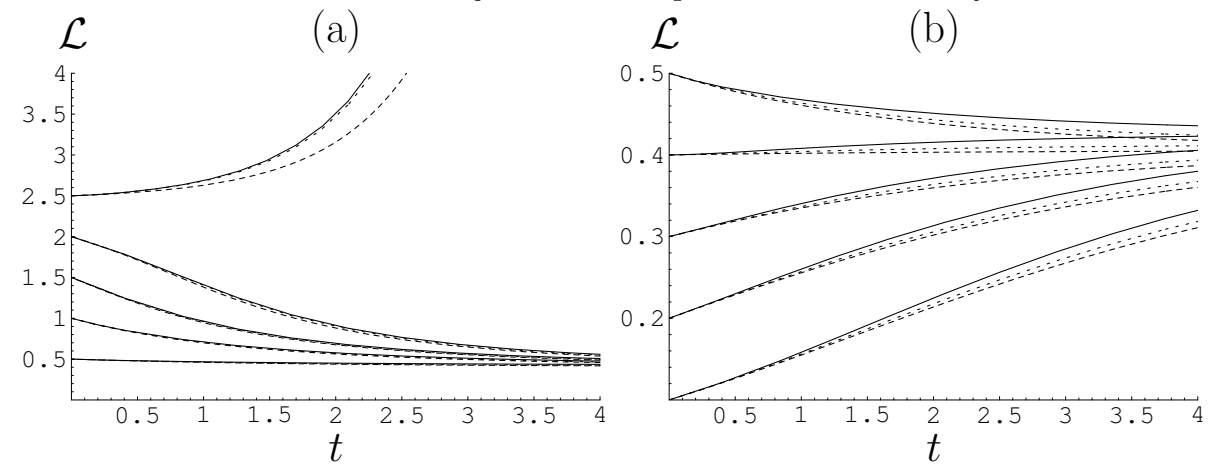

Figure 8. Dimensionless drop length $\mathcal{L}$ versus time $t$; axisymmetric solution (dashed), $m_{2}=0$ solution (solid), $m_{2}=1$ solution (dotted). Dimensionless strain rate $\mathcal{A}=0.1$ and initial length (a) $\mathcal{L}_{0}=0.5,1.0,1.5,2.0,2.5$, (b) $\mathcal{L}_{0}=0.1,0.2,0.3,0.4,0.5$.

where the dimensionless length $\mathcal{L}(t)$ satisfies the initial-value problem

$$
\frac{1}{\mathcal{L}} \frac{\mathrm{d} \mathcal{L}}{\mathrm{d} t}=1-\frac{\sqrt{5 \mathcal{L}}}{\sqrt{2} \mathcal{A}\left(10+\mathcal{L}^{3}\right)}, \quad \mathcal{L}(0)=\mathcal{L}_{0}
$$

This makes it clear, for example, that only the lower branch of steady solutions shown in figure 6 is stable.

When $\delta$ is nonzero, there is no such simple exact solution. Unlike section 4.3, we may not assume here that $\nu$ depends only on $t$, since (5.29) implies that $\nu$ must tend to zero as $x \rightarrow \ell(t)$. Instead, we follow Hinch \& Acrivos (1980) in writing $a$ and $\nu$ as truncated modal expansions in powers of $x$,

$$
a(x, t)=\left(1-\frac{x^{2}}{\ell(t)^{2}}\right) \sum_{j=0}^{m_{1}} A_{j}(t) x^{2 j}, \quad \nu(x, t)=\left(1-\frac{x^{2}}{\ell(t)^{2}}\right) \sum_{j=0}^{m_{2}} B_{j}(t) x^{2 j},
$$

where $\ell(t)$ is determined from the volume constraint (5.31). By expanding (5.28) and (5.29) up to $O\left(x^{2 m_{1}}\right)$ and $O\left(x^{2 m_{2}}\right)$ respectively we thus obtain a system of $m_{1}+m_{2}+2$ ordinary differential equations for $A_{0}(t), \ldots, A_{m_{1}}(t)$ and $B_{0}(t), \ldots, B_{m_{2}}(t)$.

In nearly all the numerical solutions shown below, we set $m_{1}=0$, which seems to give reasonable accuracy (in that including further terms in the expansion for $a$ has little effect on the solution). This presumably reflects the fact that a quadratic function of the 

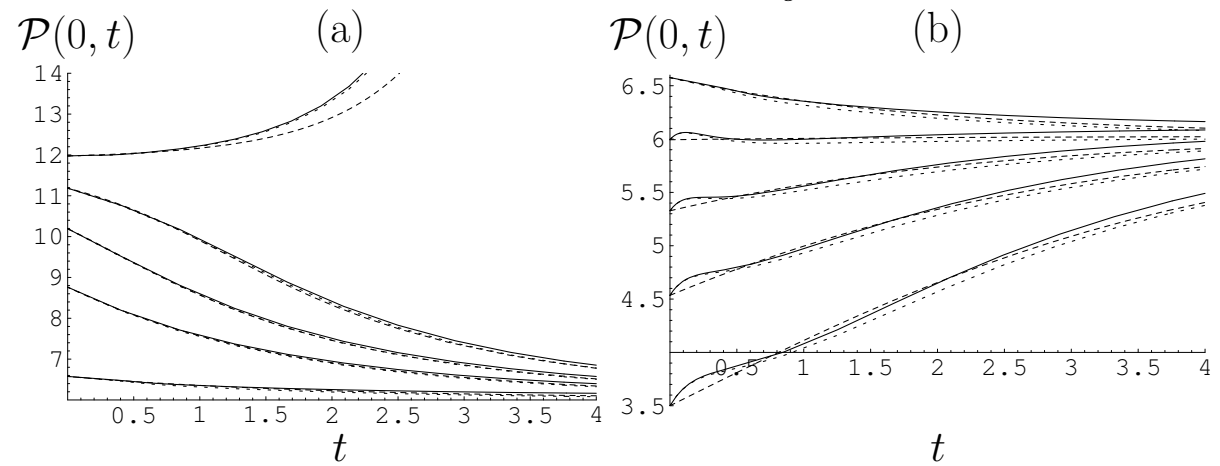

Figure 9. Pressure at the centre of the drop $\mathcal{P}(0)$ versus time $t$; axisymmetric solution (dashed), $m_{2}=0$ solution (solid), $m_{2}=1$ solution (dotted). Dimensionless strain rate $\mathcal{A}=0.1$ and initial length (a) $\mathcal{L}_{0}=0.5,1.0,1.5,2.0,2.5$, (b) $\mathcal{L}_{0}=0.1,0.2,0.3,0.4,0.5$.

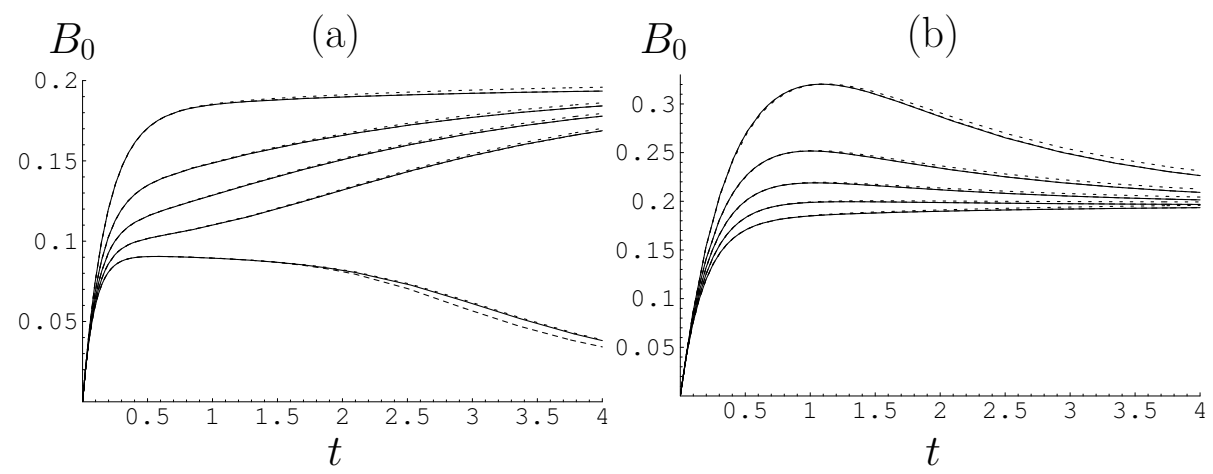

FiguRE 10. Eccentricity parameter at the drop centre $\nu(0, t)=B_{0}(t)$ versus time $t ; m_{2}=0$ solution (solid), $m_{2}=1$ solution (dotted), $m_{2}=0$ and $m_{1}=1$ solution (dashed). Dimensionless strain rate $\mathcal{A}=0.1$ and initial length (a) $\mathcal{L}_{0}=0.5,1.0,1.5,2.0,2.5$, (b) $\mathcal{L}_{0}=0.1,0.2,0.3,0.4,0.5$.
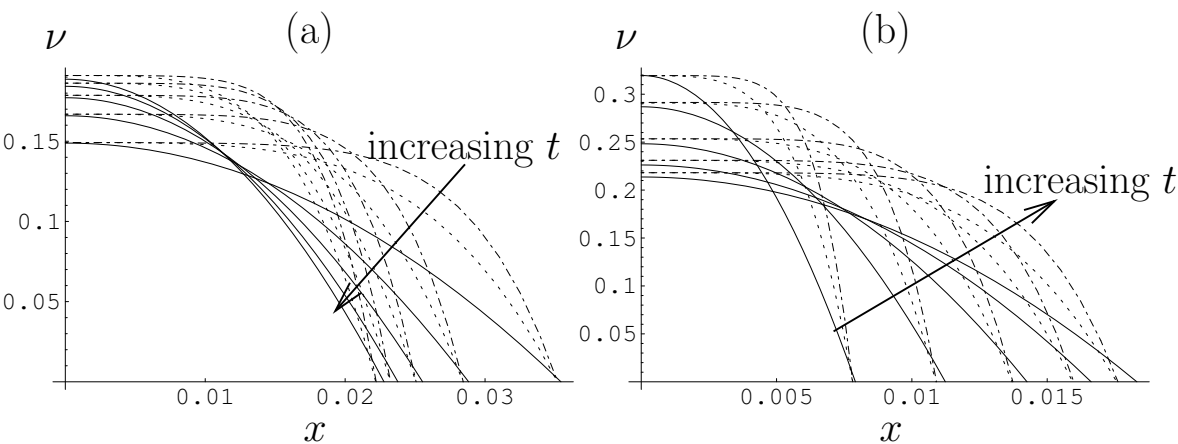

Figure 11. Eccentricity parameter $\nu$ versus $x$ for $t=1,2,3,4,5, \mathcal{A}=0.1$ and (a) $\mathcal{L}=1.0$, (b) $\mathcal{L}=0.1 ; m_{2}=0$ solution (solid), $m_{2}=1$ solution (dotted), $m_{2}=2$ solution (dot-dashed). 


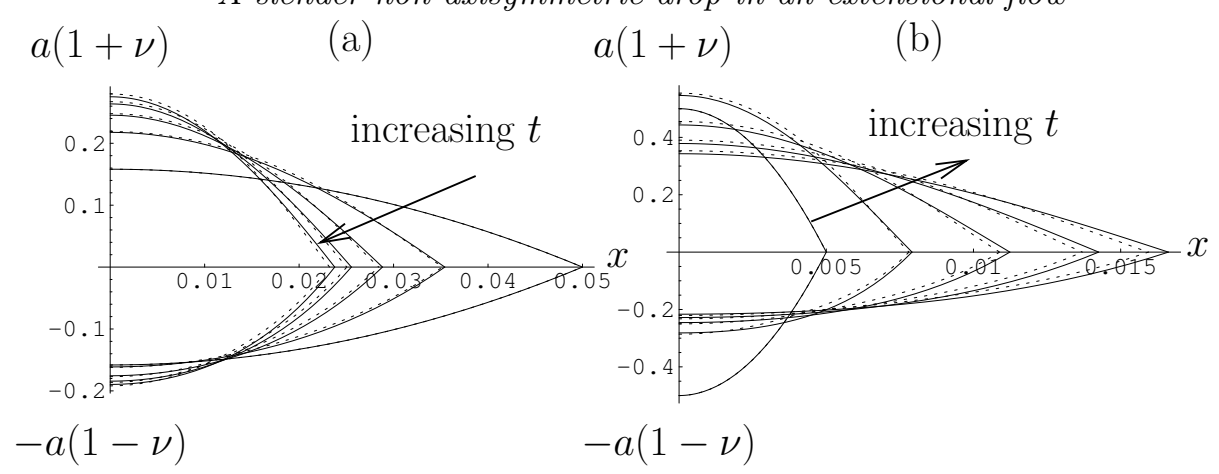

Figure 12. Drop radii $a(1+\nu)$ and $a(1-\nu)$ versus $x$ for $t=0,1,2,3,4, \mathcal{A}=0.1$ and

(a) $\mathcal{L}=1.0$, (b) $\mathcal{L}=0.1 ; m_{2}=0$ solution (solid), $m_{2}=1$ solution (dotted).

form (5.32) solves the axisymmetric problem exactly. We show solutions with $m_{2}=0$ as solid lines, $m_{2}=1$ as dotted lines and axisymmetric solutions of (5.33) as dashed lines for comparison. In all the solutions, we set $\mathcal{A}=0.1, \delta=0.5$ and experiment with various values of $\mathcal{L}_{0}$.

In figure 8 we show the evolution of the dimensionless length $\mathcal{L}$. With $\mathcal{A}=0.1$, the stable and unstable equilibrium values are $\mathcal{L} \approx 0.405$ and $\mathcal{L} \approx 2.45$ respectively. Our numerical solutions show the drop lengthening or shrinking towards its steady state depending on whether $\mathcal{L}_{0}$ is less or greater than 0.405 . The exception is $\mathcal{L}_{0}=2.5>2.45$, when the drop lengthens indefinitely, $\mathcal{L}$ growing exponentially with $t$. In all except this last case, the $m_{2}=0$ and $m_{2}=1$ solutions are close, suggesting that the solution is reasonably well captured by truncating the expansion for $\nu$ after just one term. Both solutions closely follow the axisymmetric solution, although it is noticeable in figure 8(b) that the non-axisymmetric drop approaches a slightly different equilibrium length (as indicated by figure 6 ). The unstable growing solution in figure $8(\mathrm{a})$, however, shows a quantitative difference between the one-term and two-term expansions, with $m_{2}=1$ more closely following the axisymmetric solution. This suggests that the two-term truncation performs significantly better in this case. 
In figure 9 , we show the corresponding behaviour of the pressure $\mathcal{P}(0, t)$ at the centre of the drop. In the stable drops, the pressure either increases or decreases towards its equilibrium value depending on whether $\mathcal{L}$ starts below or above 0.405 . In the increasing solutions, $\mathcal{P}$ undergoes initial transient growth similar to that seen in figure 3 . This effect is well reproduced by both the $m_{2}=0$ and $m_{2}=1$ solutions, indicating that it is not an artefact of our truncation. In the unstable drop, $\mathcal{P}$ grows exponentially and the $m_{2}=1$ solution appears to perform better, following the axisymmetric solution more closely.

Figure 10 shows the evolution of the eccentricity parameter $B_{0}=\nu(0, t)$ at the centre of the drop. Our initial condition is axisymmetric so $\nu$ starts at zero and increases towards its equilibrium value. As in figure 4, there is a significant overshoot in those cases where the drop is lengthening, and this is responsible for the initial deviations in $\mathcal{P}$ observed above. In particular, the eccentricity of the unstable drop with $\mathcal{L}_{0}=2.5$ increases initially before tending to zero as the length tends to infinity. In this figure only, we include as dashed curves the solutions with $m_{1}=1$ and $m_{2}=0$. These are everywhere very close to the $m_{1}=0, m_{2}=0$ solutions, supporting our claim that acceptable accuracy is obtained with just one term in the expansion for $a$. A noticeable, although still small, difference does occur if $m_{2}$ is increased to 1 , as shown by the dotted curves.

The variation in eccentricity over the length of the drop is shown in figure 11 for $\mathcal{A}=0.1$ and (a) $\mathcal{L}_{0}=1.0$, (b) $\mathcal{L}_{0}=0.1$. In the former case, the drop shortens with time while the eccentricity, initially set to zero, increases. In graph (b), the drop length increases with time and, as observed in figure 10(b), the eccentricity initially overshoots before relaxing to its equilibrium value. In both figures, it is clear that, although the 1-term expansion gets $\nu(0, t)$ approximately right, it fails to capture the $x$-variations in $\nu$ accurately. The profile obtained from the $m_{2}=1$ calculation is flatter, with $\nu$ almost uniform along the drop except close to the tip, and this behaviour cannot be described 
using a simple quadratic function of $x$. If one further term is kept $\left(m_{2}=2\right)$ then, as shown by the dot-dashed curves, the variations in $\nu$ are confined to an even narrower neighbourhood of the tip.

It is fortunate that quantities of interest, such as $\mathcal{L}$ and $\mathcal{P}$, are not unduly affected by the accuracy with which the $x$-dependence of $\nu$ is resolved. For example, in figure 12 we show how typical profiles of the drop radii $a(1 \pm \nu)$ vary with time, starting from (a) $\mathcal{L}_{0}=1.0$ (so the drop length decreases with $t$ ), (b) $\mathcal{L}_{0}=0.1$ (so the drop length increases with $t$ ). In either case, the initial state is axisymmetric but, as $t$ increases, the difference between the two radii becomes apparent. Despite the discrepancy in the behaviour of $\nu$ shown in figure 11, the gross features of the drop geometry are captured well by the $m_{2}=0$ solution. The errors in $\nu$ are greatest near the tip, where $a$ is small so, on the scale of the whole drop, they make relatively little difference.

\section{Slender jets}

\subsection{Problem description}

The equations derived thus far also describe a jet of fluid in a much more viscous external fluid. For example, Doshi et al. (2003) describe the rupture of a low-viscosity drop in a viscous fluid. The rupture occurs via the formation of a thin thread of relatively inviscid fluid, which then breaks up due to a surface-tension-driven instability. Our model equations apply to the dynamics of such a thread. Equally, one can imagine shooting a jet of fluid (say air) into one of much higher viscosity (say golden syrup); the evolution of such a jet is also governed by our equations.

We consider the model problem of a periodic jet subject to a purely two-dimensional straining flow perpendicular to the axis of the jet. This corresponds to taking the limit 
$\mathrm{C} \rightarrow 0$ in (4.13), (4.14) and (5.15), while keeping $\delta \mathrm{C}$ finite. Thus we obtain the model

$$
\begin{aligned}
& a_{t}=\frac{1}{2} \mathcal{P} a-k(\nu), \\
& \nu_{t}=2 \mathcal{C}-\mathcal{P} \nu, \\
&\left(\frac{\left(1-\nu^{2}\right)^{3}}{\left(1+\nu^{2}\right)} a^{4} \mathcal{P}_{x}\right)_{x}=8 \lambda a\left(\left(1+\nu^{2}\right) \mathcal{P} a-4 \mathcal{C} \nu a-2\left(1-\nu^{2}\right) k(\nu)\right),
\end{aligned}
$$

where $\mathcal{C}=\delta \mathrm{C}$, with boundary and initial conditions

$$
\begin{array}{rl}
\mathcal{P}_{x}=0 & x=0, x=\pi, \\
a=a_{0}(x), \nu=\nu_{0}(x) & t=0 .
\end{array}
$$

Integration of $(6.1 c)$ with respect to $x$ leads to the identity

$$
\frac{\mathrm{d}}{\mathrm{d} t} \int_{0}^{\pi} a^{2}\left(1-\nu^{2}\right) \mathrm{d} x=0,
$$

corresponding to net conservation of mass, so we can non-dimensionalise such that

$$
\int_{0}^{\pi} a^{2}\left(1-\nu^{2}\right) \mathrm{d} x=\pi
$$

\subsection{Axisymmetric solutions}

We start by considering the axisymmetric case, in which $\mathcal{C}$ and $\nu$ are both identically zero, so (6.1) reduces to

$$
\begin{aligned}
a_{t} & =\frac{1}{2}(\mathcal{P} a-1), \\
\left(a^{4} \mathcal{P}_{x}\right)_{x} & =8 \lambda a(\mathcal{P} a-1) .
\end{aligned}
$$

In figure 13 we show a typical numerical solution of (6.5) subject to the boundary conditions (6.2) with $\lambda=1$ and initial data

$$
a_{0}(x)=c_{0}+c_{1} \cos x, \quad c_{1}=0.05, c_{0}=\sqrt{1-c_{1}^{2} / 2}
$$

where the latter expression follows from the requirement (6.4) with $\nu=0$. The small perturbation initially grows slowly until $t \sim 30$, when $a$ starts to become small enough for surface tension effects to be significant. Thereafter, the minimum point rapidly decreases, 


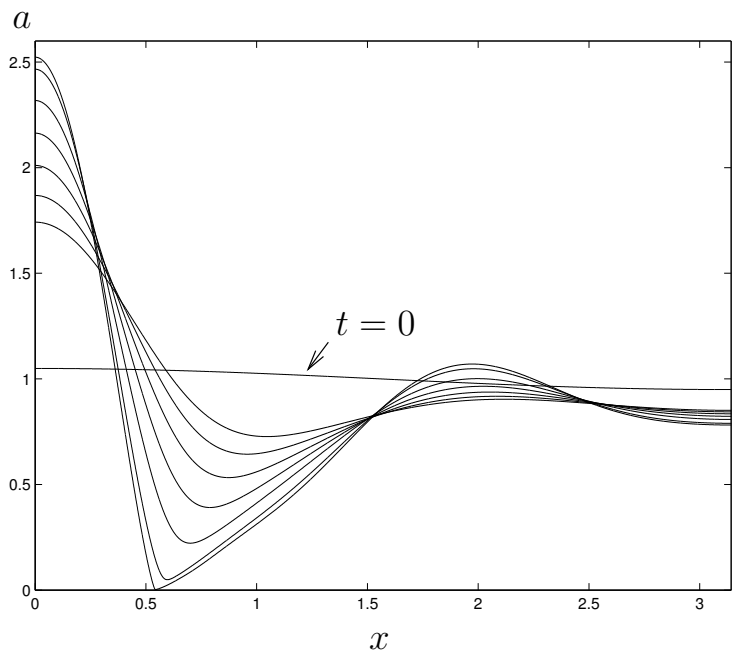

FIGURE 13. Numerical solution of axisymmetric equations (6.5) with $\lambda=1$, $a(x, 0)=c_{0}+c_{1} \cos x, c_{1}=0.05$ and $c_{0}=\left(1-c_{1}^{2} / 2\right)^{1 / 2} ; t=0,30,31,32,33,34,35,35.4$. appearing to reach zero at a finite time $t \approx 35.4$. The self-similar behaviour of $(6.5)$ as $a \rightarrow 0$, showing that the jet does indeed pinch off in finite time, has been obtained by Sierou \& Lister (2003).

\subsection{Non-axisymmetric solutions}

Next we present numerical solutions of the non-axisymmetric model (6.1). First we set $\mathcal{C}=0.25$ and initialise the problem with

$$
\nu(x, 0)=\nu_{0}=0.5, \quad a_{0}(x)=c_{0}+c_{1} \cos x, \quad c_{1}=0.1, \quad c_{0}=\sqrt{\left(1-\nu_{0}\right)^{-1}-c_{1}^{2} / 2}
$$

the latter relation again following from (6.4). The resulting evolutions of the two principle radii are shown in figure 14 . The behaviour is similar to the axisymmetric solution shown in figure 13: both radii tend to zero at the same finite time, so the jet pinches off at a point. Indeed, from (6.1) one may readily deduce that, if $a$ is to approach zero, then $\nu$ must do so also. The local behaviour near pinch-off must therefore be axisymmetric (as is exhibited by the simulations) and, hence, described by the self-similar solutions obtained by Sierou \& Lister (2003). 

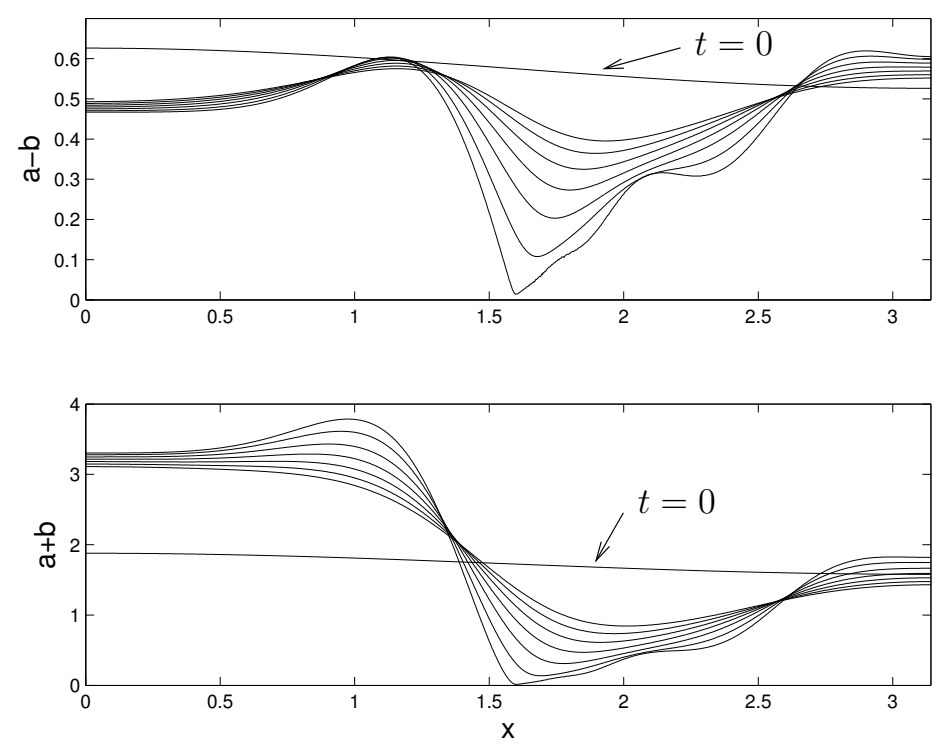

FIgURE 14. Numerical solution of the non-axisymmetric equations (6.1) with $\mathcal{C}=0.25$, $\lambda=1, \nu_{0} \equiv 0.5, a(x, 0)=c_{0}+c_{1} \cos x, c_{1}=0.1$ and $c_{0}=\left[\left(1-\nu_{0}\right)^{-1}-c_{1}^{2} / 2\right]^{1 / 2} ;$ $t=0,38,39,40,41,42,43,43.8$.

In contrast, consider the simulation shown in figure 15 , in which $\mathcal{C}=0.35, \lambda=1$, $\nu_{0}=0.8$ and $a_{1}=0.1$. Now the jet does not appear to pinch off at all. Instead it is squashed by the applied straining flow, such that one principal radius tends to infinity while the other tends to zero.

In the light of these sample simulations, it is natural to ask what determines whether a given set of initial data results in finite-time pinch-off or in lateral stretching of the jet. A partial answer to this question may be obtained as follows. Steady, uniform solutions of (6.1) must satisfy

$$
\mathcal{C} a=\nu k(\nu)=\frac{1}{2} \mathcal{P} \nu a
$$

By enforcing the constraint (6.4), we obtain the following relation between the applied strain rate $\mathcal{C}$ and the eccentricity parameter $\nu$ :

$$
\mathcal{C}=\nu k(\nu) \sqrt{1-\nu^{2}}
$$



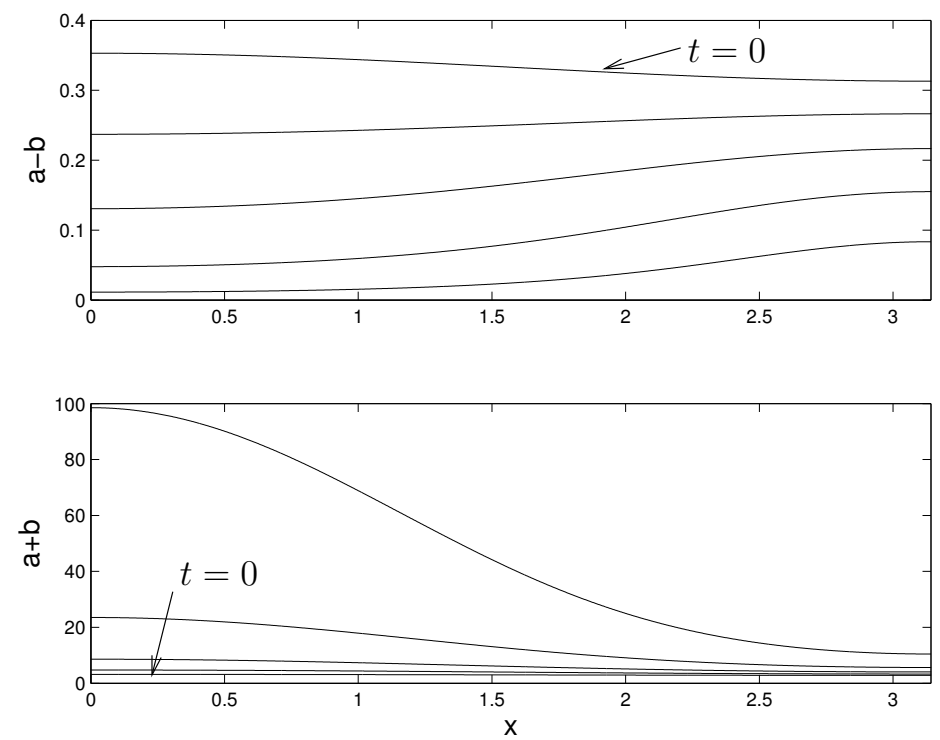

FigURE 15. Numerical solution of the non-axisymmetric equations $(6.1)$ with $\mathcal{C}=0.35, \lambda=1$, $\nu_{0} \equiv 0.8, a(x, 0)=c_{0}+c_{1} \cos x, c_{1}=0.1$ and $c_{0}=\left[\left(1-\nu_{0}\right)^{-1}-c_{1}^{2} / 2\right]^{1 / 2} ; t=0,5,10,15,20$.

This relation, illustrated in figure 16, has qualitative behaviour similar to that for the axisymmetric problem shown in figure 6 . If the applied strain rate $\mathcal{C}$ exceeds a critical value $\mathcal{C}^{*} \approx 0.30486$, then there is no uniform elliptical solution while, for $\mathcal{C}<\mathcal{C}^{*}$, there are two possible values of $\nu$, of which the lower is stable.

Although the general behaviour of spatially and temporally non-uniform solutions of (6.1) depends on the initial conditions applied, some useful insight may be gained from figure 16. For $\mathcal{C}$ less than the critical value $\mathcal{C}^{*}$, the lower branch gives two-dimensional steady solutions that are unstable to perturbations in the $x$-direction and, presumably, lead to break-up of the jet. On the other hand, if $\mathcal{C}>\mathcal{C}^{*}$, or $\nu$ exceeds the unstable upper branch in figure 16, then there is no steady two-dimensional solution so the jet presumably stretches under the straining flow rather than breaking up. This picture of the solution structure agrees with the simulations shown in figures 14 and 15, although it would clearly be possible to construct initial conditions such that part of the jet is in the pinching regime while the rest is in the stretching regime. 


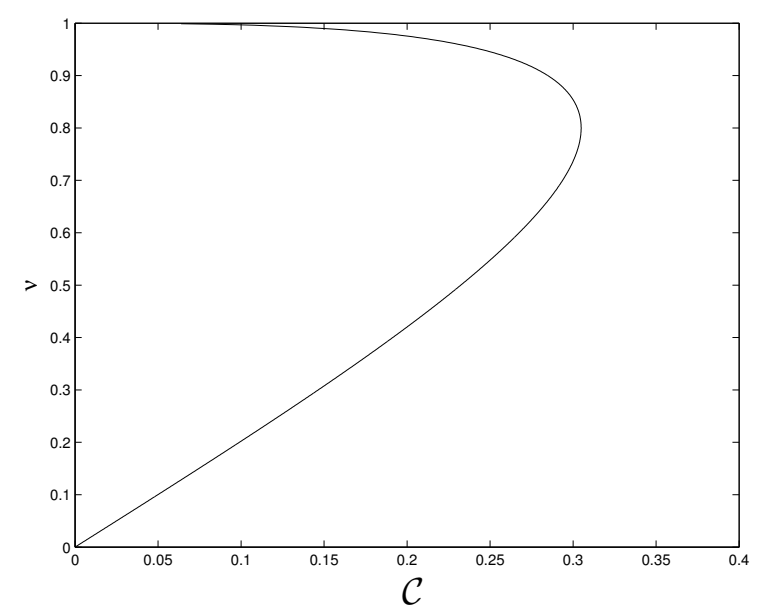

FiguRE 16. Bifurcation diagram of $\nu$ versus $\mathcal{C}$ for uniform solutions of (6.1).

\section{Conclusions}

In this paper we have analysed the behaviour of a slender drop or jet in a straining flow of a much more viscous fluid. This scenario has previously been studied in some detail in two special cases. The first, in which the flow is axisymmetric, was described by Taylor (1964); Buckmaster $(1972,1973)$. The second, in which the flow is purely two-dimensional, was first considered in the steady state by Richardson (1968) and subsequently by Tanveer \& Vasconcelos (1995). These authors give a method for obtaining exact solutions of the two-dimensional Stokes equations, with surface tension on the bubble surface. Our governing equations represent a combination of the two approaches, and allow us to describe jets that are neither two-dimensional nor axisymmetric. Our study is limited to elliptical cross-sections, exploiting the property noted by Crowdy (2003) that a twodimensional compressible bubble that is initially elliptical remains so when deformed by a linear flow. Our model thus consists of a system of partial differential equations (4.13), (4.14) and (5.15) for the average radius $a$ and shape parameter $\nu$ (related to the eccentricity) of each cross-section, and the pressure $\mathcal{P}$ inside the drop.

For a bubble containing inviscid fluid, the eccentricity was found to be spatially uni- 
form. This enabled us to obtain explicit solutions for steady non-axisymmetric bubbles and to reduce the unsteady problem to a system of two nonlinear ordinary differential equations. In either case, we confirmed the result of Hinch \& Acrivos (1979) that two-dimensional straining flow may safely be viewed as a small perturbation of the corresponding axisymmetric flow. Our simulations considered an initially axisymmetric drop in a two-dimensional extensional flow, which may be viewed as an impulsive change in the external flow from axisymmetric to two-dimensional. The resulting adjustment in the cross-section eccentricity gives rise to noticeable pressure fluctuations not exhibited by purely axisymmetric bubbles.

For a drop containing slightly viscous fluid, we obtained numerical steady solutions that agreed closely with the axisymmetric solutions found previously by Taylor (1964); Acrivos \& Lo (1978), except that the unstable branch of the bifurcation diagram in figure 7 is significantly altered. This was shown to be due to the non-axisymmetric solution switching to an alternative solution branch found previously by Buckmaster (1973). We also obtained approximate unsteady solutions based on writing the drop radius and eccentricity as truncated polynomial expansions. As for inviscid bubbles, we found that the evolutions of the drop length and pressure are well approximated by axisymmetric solutions, except for small pressure variations caused by changes in the cross-section eccentricity. In both cases, though, the drop geometry is in fact quite far from being axisymmetric, as shown in figure 12 for example.

We have only considered a drop whose initial radius has a convex quadratic profile. Our approach could, however, easily be generalised to a drop with a local minimum which would, as shown by Hinch (1980), be expected to pinch off in finite time. Instead, we applied our theory to a periodic slender jet inside a much more viscous fluid. We found that the universal pinching behaviour exhibited by axisymmetric jets (Sierou \& Lister 
2003) may be prevented by applying of a suitable transverse straining flow. Instead of the jet pinching off in finite time, its two principal radii tend to zero and infinity respectively, while their product stays finite. This behaviour suggests that an appropriately modulated straining flow may stabilise the jet against pinch-off.

Our analysis relies on using the slenderness of the geometry to reduce the threedimensional flow problem to a sequence of two-dimensional problems. It is worth pointing out that a similar reduction in dimension may be achieved in the opposite limit, in which the bubble radius varies sufficiently rapidly for the azimuthal component of curvature to be negligible (Eggers, Lister \& Stone 1999).

Although we have limited our attention to the simple linear extensional flow (2.2), many other flows are amenable to similar analysis. Hinch \& Acrivos (1980), for example, considered the deformation of a slender drop in shear flow, under the ad hoc assumption that the cross-section is approximately circular. Our approach should enable the accuracy of this approximation to be explored further. Nonlinear external flows, considered in the axisymmetric case by Sherwood (1984), may also be analysed using our approach by exploiting the two-dimensional solutions of Antanovskii (1996) and Siegel (2000).

The methods discussed here may be useful in applications such as modelling the drawing of microstructured optical fibres, for example those whose cross-sections contain an array of holes spanning the length of the fibre. The technological importance of these fibres lies in their ability to guide light, due to the jump in refractive index between the solid core and air-hole laced cladding. A theoretical model of the drawing process has been provided by Fitt et al. (2001, 2002), although the assumption there of axisymmetry precludes the consideration of multiple-holed structures. The methods described here can be extended to treat cross-sections with multiply connected fluid regions by utilis- 
ing the techniques developed in Crowdy \& Tanveer (1998) and Richardson (2000) (and generalized in Crowdy 2002). We intend to explore such an extension in future work.

\section{Acknowledgements}

The work of one of the authors (Siegel) was partially performed while visiting the Oxford Centre for Industrial and Applied Mathematics (OCIAM). Their hospitality and support is gratefully acknowledged. Additional support has been provided by NSF Grant DMS-0104350 (Siegel).

\section{REFERENCES}

Acrivos, A. \& Lo, T. S. 1978 Deformation and breakup of a single slender drop in an extensional flow. J. Fluid Mech. 86, 641-672.

Antanovskit, L. K. 1994 Quasi-steady deformation of a two-dimensional bubble placed within a potential viscous flow. Meccanica-J. Ital. Assoc. Thoert. Appl. Math. 29, 27-42.

AntanovskiI, L. K. 1996 Formation of a pointed drop in Taylor's four-roller mill. J. Fluid Mech. 327, 325-341.

Buckmaster, J. D. 1972 Pointed bubbles in slow viscous flow. J. Fluid Mech. 55, 385-400.

Buckmaster, J. D. 1973 The bursting of pointed drops in slow viscous flow. Trans. A.S.M.E. E, J. Appl. Mech. 40, 18-24.

Crowdy, D. G. 2002 Exact solutions for the viscous sintering of multiply-connected fluid domains. J. Eng. Math. 42, 225-242.

Crowdy, D. G. 2003 Compressible bubbles in Stokes flow. J. Fluid Mech. 476, 345-356.

Crowdy, D. G. \& Tanveer, S. 1998 A theory of exact solutions for annular viscous blobs. $J$. Nonlinear Sci. 8, 375-400.

Cummings, L. J. \& Howell, P. D. 1999 On the evolution of non-axisymmetric viscous fibres with surface tension, inertia and gravity. J. Fluid Mech. 389, 361-389.

Doshi, P., Cohen, I., Zhang, W. W., Siegel, M., Howell, P. D., Basaran, O. A. \& 
NAGEL, S. R. 2003 Persistence of memory in drop breakup: The breakdown of universality. Science 302, 1185-1188.

Eggers, J., Lister, J. R. \& Stone, H. A. 1999 Coalescence of liquid drops. J. Fluid Mech. 401, 293-310.

Fitt, A. D., Furusawa, K., Monro, T. M. \& Please, C. P. 2001 Modeling the fabrication of hollow fibres: Capillary drawing. J. Lightwave Technol. 19(12) 1924-1931.

Fitt, A. D., Furusawa, K., Monro, T. M., Please, C. P. \& Richardson, D. J. 2002 The mathematical modelling of capillary drawing for holey fibre manufacture. J. Eng. Maths 43, 201-227.

Gradshteyn, I. S. \& Ryzhik, I. M. 1994 Table of Integrals, Series, and Products. Fifth Edition. Academic Press.

Hinch, E. J. 1980 The evolution of slender inviscid drops in an axisymmetric straining flow. J. Fluid Mech. 101, 545-553.

Hinch, E. J. \& ACRIVos, A. 1979 Steady long slender droplets in two-dimensional straining motion. J. Fluid Mech. 91, 401-414.

Hinch, E. J. \& Acrivos, A. 1980 Long slender drops in a simple shear flow. J. Fluid Mech. 98, 305-328.

Howell, P. D. 1994 Extensional Thin Layer Flows. D. Phil. Thesis, University of Oxford.

PozRikidis, C. 2001 Expansion of a compressible gas bubble in Stokes flow. J. Fluid Mech.442, 171-189.

Richardson, S. 1968 Two-dimensional bubbles in slow viscous flows. J. Fluid. Mech. 33, 476493.

Richardson, S. 2000 Plane Stokes flow with time-dependent free boundaries in which the fluid occupies a doubly-connected region. Eur. J. Appl. Math. 11, 249-269.

Sherwood, J. D. 1984 Tip streaming from slender drops in a nonlinear extensional flow. J. Fluid Mech. 144, 281-295.

Siegel, M. 2000 Cusp formation for time-evolving bubbles in two-dimensional Stokes flow. J. Fluid Mech. 412, 227-257. 
Sierou, A. \& Lister, J. R. 2003 Self-similar solutions for viscous capillary pinch-off. J. Fluid Mech. 497, 381-403.

Stone, H. A. 1994 Dynamics of drop deformation and breakup in viscous fluids. Ann. Rev. Fluid Mech. 26, 65-102.

Tanveer, S. \& Vasconcelos, G. L. 1995 Time-evolving bubbles in 2-dimensional Stokes-flow. J. Fluid Mech. 301, 325-344.

TAYLOR, G. I. 1934 The formation of emulsions in definable fields of flow. Proc. Roy. Soc. A 146, 501-523.

TAYlor, G. I. 1964 Conical free surfaces and fluid interfaces. Proc. 11th Int. Cong. Appl. Mech. (Munich), Springer-Verlag, 790-796.

Zhang, W. W. \& Lister, J. R. 1999 Similarity solutions for capillary pinch-off in fluids of differing viscosity. Phys. Rev. Lett. 83(6), 1151-1154. 